\title{
Frequency-specific network topologies in the resting human brain
}

\section{Shuntaro Sasai ${ }^{1,2 *}$, Fumitaka Homae ${ }^{3}$, Hama Watanabe ${ }^{1}$, Akihiro T. Sasaki $^{4,5}$, Hiroki C. Tanabe ${ }^{6}$, Norihiro Sadato ${ }^{78}$ and Gentaro Taga ${ }^{1}$}

${ }^{1}$ Graduate School of Education, University of Tokyo, Tokyo, Japan

2 Department of Psychiatry, University of Wisconsin - Madison, Madison, WI, USA

${ }^{3}$ Department of Language Sciences, Tokyo Metropolitan University, Tokyo, Japan

${ }^{4}$ Pathophysiological and Health Science Team, Imaging Application Group, Division of Bio-function Dynamics Imaging, RIKEN Center for Life Science Technologies, Kobe, Japan

${ }^{5}$ Department of Physiology, Osaka City University Graduate School of Medicine, Osaka, Japan

${ }^{6}$ Graduate School of Environmental Studies, Nagoya University, Nagoya, Japan

${ }^{7}$ Division of Cerebral Integration, Department of Cerebral Research, National Institute for Physiological Sciences, Okazaki, Japan

${ }^{8}$ Department of Physiological Sciences, SOKENDAI (The Graduate University for Advanced Studies), Okazaki, Japan

\section{Edited by:}

Shuhei Yamaguchi, Shimane

University, Japan

\section{Reviewed by:}

Hellmuth Obrig, Max Planck

Institute of Human Cognitive and

Brain Sciences, Germany

Keiichi Kitajo, RIKEN Brain Science

Institute, Japan

*Correspondence:

Shuntaro Sasai, Department of

Psychiatry, University of Wisconsin -

Madison, 6001 Research Park, Blvd.,

Madison, WI 53719, USA

e-mail: sasai@p.u-tokyo.ac.jp
A community is a set of nodes with dense inter-connections, while there are sparse connections between different communities. A hub is a highly connected node with high centrality. It has been shown that both "communities" and "hubs" exist simultaneously in the brain's functional connectivity network (FCN), as estimated by correlations among low-frequency spontaneous fluctuations in functional magnetic resonance imaging (fMRI) signal changes $(0.01-0.10 \mathrm{~Hz})$. This indicates that the brain has a spatial organization that promotes both segregation and integration of information. Here, we demonstrate that frequency-specific network topologies that characterize segregation and integration also exist within this frequency range. In investigating the coherence spectrum among 87 brain regions, we found that two frequency bands, $0.01-0.03 \mathrm{~Hz}$ (very low frequency [VLF] band) and $0.07-0.09 \mathrm{~Hz}$ (low frequency [LF] band), mainly contributed to functional connectivity. Comparing graph theoretical indices for the VLF and LF bands revealed that the network in the former had a higher capacity for information segregation between identified communities than the latter. Hubs in the VLF band were mainly located within the anterior cingulate cortices, whereas those in the LF band were located in the posterior cingulate cortices and thalamus. Thus, depending on the timescale of brain activity, at least two distinct network topologies contributed to information segregation and integration. This suggests that the brain intrinsically has timescale-dependent functional organizations.

Keywords: resting-state fMRI, frequency-dependency, network analysis, functional connectivity, rich-club connectivity, community, integration, segregation

\section{INTRODUCTION}

Functional connectivity indicates statistical dependency with the activity between brain regions, implying that they share information. Since similar spatial characteristics of functional connectivity have been demonstrated during both task execution and rest, the brain might not be a stimulus-responsive organ. Rather, it might function through the intrinsic activity constraints (Biswal et al., 1995; Fox and Raichle, 2007). Functional magnetic resonance imaging (fMRI) has been used to investigate functional connectivity across the whole brain; related findings have provided a fundamental view of the brain's spatial organization that simultaneously achieves information segregation and integration (Sporns, 2013). The brain has a characteristic network structure that promotes independence between clusters of regions, as well as enhances inter-dependence across all areas. Furthermore, fMRI signals contain multiple time-scale components; the coexistence of fMRI signals fluctuating at several time scales has been shown (Zuo et al., 2010; Baria et al., 2011; He, 2011). However, the relevance of these time-scale components to the spatial architecture of functional connectivity is unclear. Therefore, the current study investigated frequency specificity of the brain's functional connectivity profile that contributes to information segregation and integration.

Spatial organization of spontaneous brain activity has been studied from the viewpoint of graph theory. Based on this theory a functional connectivity network (FCN) is constructed by viewing functional connectivity as an edge, and each brain region as a node. The FCN also has sub-network structures consisting of densely interconnected regions called "modules" or "communities." Recent studies have provided evidence suggesting that different communities are less correlated to each other and have own differentiated functions (Dosenbach et al., 2007, 2010; Power et al., 2011; Fornito et al., 2012; Spreng et al., 2013). Conversely, highly connected central regions are called "hubs" (Achard et al., 2006; van den Heuvel et al., 2008b; Buckner et al., 2009; Tomasi and Volkow, 2011a,b). Hubs are thought to be important for 
integration in FCN by bridging between different communities via those called "connectors" (Sporns et al., 2007; Hagmann et al., 2008; Meunier et al., 2009; Power et al., 2013), and by forming a higher-order structure, referred to as "rich-club" with dense interconnections (Crossley et al., 2013; Grayson et al., 2014). The presence of these network structures indicates that FCN has a topology enabling the network to concurrently segregate and integrate information.

It has been demonstrated that fMRI signals contributing to functional connectivity exhibit "low-frequency fluctuations" within $0.01-0.10 \mathrm{~Hz}$ (Cordes et al., 2001). Therefore, most fMRI studies on network-level organization of functional connectivity have focused on synchronized low-frequency fluctuations of fMRI signal changes $(0.01-0.10 \mathrm{~Hz}$ ) in the resting brain (Fox and Raichle, 2007). However, a computational study demonstrated that topological features of functional connectivity could vary with the timescale of brain activity without changing the underlying structural connections (Honey et al., 2007). Indeed, frequency-specific characteristics exist in correlations with hemodynamic fluctuation within this low-frequency range, and differ depending on the particular combination of brain regions (Wu et al., 2008; Chang and Glover, 2010; Sasai et al., 2011). Furthermore, an fMRI study has demonstrated that some brain regions not only show event-related activity occurring at typical timescales for hemodynamic responses to a single event $(0.05-0.10 \mathrm{~Hz})$, but also display signal increases that are sustained for the duration of a task block (Dosenbach et al., 2006). These studies suggest that FCN derived by from fMRI could have frequency-specific topologies that potentially have different functional relevance with specific information structures; however, to our knowledge, no previous report exists regarding his issue. Thus, the present study investigated the relationship between different frequency components of spontaneous fMRI signal fluctuations and network structures responsible for information segregation and integration in FCN.

\section{MATERIALS AND METHODS PARTICIPANTS}

A total of 28 healthy adults (15 men and 13 women; age range, 22-44 years) participated in this study. The protocol was approved by the ethical committee of the National Institute for Physiological Sciences, Okazaki, Japan. Informed consent was obtained from all participants prior to taking part in the study.

\section{DATA ACOUISITION}

All MRI data used in the current study were obtained during simultaneous near-infrared spectroscopy (NIRS). All participants were instructed to remain awake with their eyes closed during data acquisition, and confirmed after the process that they had not fallen asleep. These data were similar to those used in a previous study (Sasai et al., 2012). The following sections briefly introduce the method used to obtain MRI and NIRS data in the previous study.

\section{MRI}

Structural and functional volumes were acquired using a 3-Tesla MR scanner (Allegra; Siemens). First, a time-series of 610 volumes was acquired for each session using a $\mathrm{T} 2{ }^{*}$-weighted gradient-echo echo-planar imaging (EPI) sequence. Each volume consisted of 34 slices, each of which was $3.5-\mathrm{mm}$ thick with a $17 \%$ gap. The time interval between two successive acquisitions of the same slice (TR) was $2000 \mathrm{~ms}$, with a flip angle (FA) of $76^{\circ}$, and an echo time (TE) of $30 \mathrm{~ms}$. The field of view $(\mathrm{FoV})$ was $192 \times 192 \mathrm{~mm}$, and the in-plane matrix size was $64 \times 64$ pixels. Additionally, to acquire a fine structural wholebrain image, magnetization-prepared rapid-acquisition gradientecho (MP-RAGE) images were obtained $(T R=2500 \mathrm{~ms} ; T E=$ $4.38 \mathrm{~ms} ; F A=8^{\circ} ; \mathrm{FoV}=230 \times 230 \mathrm{~mm}$; one slab; number of slices per slab $=192$; voxel dimensions $=0.9 \times 0.9 \times 1.0 \mathrm{~mm}$ ).

\section{NIRS}

We used a near-infrared optical topography instrument (ETG4000; Hitachi Medical Corporation, Tokyo, Japan) to measure the time series of spontaneous changes in oxygenated and deoxygenated hemoglobin concentrations with a 0.1 -s time resolution. The instrument generated two wavelengths of near-infrared light (695 and $830 \mathrm{~nm}$ ). We evaluated relative changes in the oxygenated and deoxygenated hemoglobin signals from an arbitrary baseline (set to 0 ) at the beginning of the measurement period based on the Lambert-Beer law. The unit used to measure these values was molar concentration multiplied by length ( $\mathrm{mM} \cdot \mathrm{mm}$ ). The distance between the incident and the detection fibers was $3 \mathrm{~cm}$. The eight emitters and eight detectors were plugged into a holder, to which vitamin tablets were attached to identify the positions of NIRS channels in MRI images, and were arranged into two $1 \times 8$ arrays, resulting in 14 measurement channels. Arrays were positioned over the bilateral frontal, temporal, and occipital regions by referring to the international 10-20 System of Electrode Placement. NIRS data were simultaneously obtained with fMRI imaging for all participants, with each participant lying supine in an MRI scanner.

\section{fMRI PREPROCESSING}

Functional MR volumes were motion-corrected and slice-timingcorrected using the SPM8 package (Wellcome Department of Imaging Neuroscience, London, UK). fMRI data sets were spatially smoothed with a 5-mm full-width-at-half-maximum Gaussian blur, and were normalized to the MNI space using DARTEL in SPM8. fMRI data sets are generally contaminated with noise, including fluctuations due to scanner instabilities, subject motion, and respiration and cardiac effects, resulting in coherent signal fluctuations across the brain (e.g., global signals). In many studies, these contaminating signals are estimated by utilizing fMRI data-inherent information, and removed using a general linear model (GLM) technique (Fox et al., 2005). However, the regression of global signal has been shown to introduce spurious anti-correlations (Murphy et al., 2009; Anderson et al., 2011). Anderson et al. (2011) have proposed an alternate method for avoiding this bias in correlation estimation; it uses an optimally phase-shifted waveform extracted from soft tissues of the face and calvarium, as well as regressors obtained from subject motion parameters, white matter, ventricles, and physiological waveforms, and is termed phase-shifted soft-tissue correction [PSTCor]. Based on the method proposed by Anderson et al. (2011), we previously used a modified version of the PSTCor that only employed fMRI inherent information, and observed no 
anti-correlation (Sasai et al., 2012). The present study applied this modified method of PSTCor to eliminate noise.

\section{ROI SELECTION}

We refer to network of the brain as "FCN," while we call its sub-structure like default mode system as a "functional system" hereafter. It has been suggested that spontaneous brain activity is organized into two widespread functional systems in terms of the activity profiles recruited by cognitively demanding tasks: "taskpositive systems" and "task-negative systems (Fox et al., 2005)." Although several studies have consistently reported activation within regions including the dorsal anterior cingulate cortex, frontal insula, lateral prefrontal cortex, and lateral parietal cortex during attention and working memory tasks (Menon et al., 2001; Curtis and D'Esposito, 2003; Kerns et al., 2004; Ridderinkhof et al., 2004; Fox et al., 2005), reduced activity in in brain regions such as the medial prefrontal cortex, angular gyrus, and posterior cingulate cortex have also been observed during these tasks (Gusnard et al., 2001; Raichle et al., 2001; Fox et al., 2005). Recently, it has been shown that the task-positive system consists of at least two different sets of brain regions in terms of its functions: central executive and saliency systems (Seeley et al., 2007; Menon and Uddin, 2010). Furthermore, Dosenbach et al. (2006) demonstrated that the task-positive system is composed of multiple sub-systems, including the fronto-parietal system overlapping with central executive systems, and the cingulo-opercular system overlapping with the saliency system, whereas the tasknegative system is composed of a single system (the default mode system). In order to investigate the existence of frequencyspecific topology in a large-scale FCN, we selected the following three functional systems, which included hub regions from both task-positive and task-negative systems: the default mode system (DMS), the fronto-parietal task control system (FPS), and the cingulo-opercular task control system (COS) (Power et al., 2011). Dosenbach et al. (2010) had identified coordinates corresponding to these functional systems by conducting a meta-analysis of the relevant literature. We used the reported coordinates to extract the time series corresponding to the functional systems. These ROIs were located in areas of the cerebral cortex and sub-cortical regions. The total number of ROIs was 87 . Table 1 summarizes the MNI coordinates, originally assigned functional systems, and names of automated anatomical labeling (AAL) of the ROIs (Tzourio-Mazoyer et al., 2002).

\section{DETECTION OF FREQUENCY SPECIFICITY FOR FUNCTIONAL CONNECTIVITY}

For each individual data set, we calculated the coherence between all pairs of signals extracted from the above-mentioned 87 ROIs with radii of $6 \mathrm{~mm}$. Coherence measures the linear and timeinvariant relationship between two signals at frequency $\lambda$ and is defined as follows:

$$
C_{x y}(\lambda)=\frac{\left|P_{x y}(\lambda)\right|^{2}}{P_{x x}(\lambda) P_{y y}(\lambda)},
$$

where $C_{x y}(\lambda)$ refers to the coherence between signals $x$ and $y$, $P_{x y}(\lambda)$ is the cross-spectrum of $x$ and $y, P_{x x}(\lambda)$ is the power spectrum of $x$, and $P_{y y}(\lambda)$ is the power spectrum of $y$. For each pair of signals, we obtained coherence matrices by averaging the coherence values within 23 narrow, 50\%-overlapping frequency bands, with bandwidths of $0.02 \mathrm{~Hz}$ (Figure 1A). Chang and Glover (2010) showed that the frequency dependency of coherence among the ROIs organizing the DMS is different from those estimated based on ROIs of two distinct functional systems (DMS and dorsal attention systems). Taking this into consideration, we further averaged these band-averaged coherence values within two categories of ROI pairs in order to identify the frequency-dependency of functional connectivity: ROIs within in the same functional system (intra-system), and ROIs belonging to different functional systems (inter-system; Dosenbach et al., 2010; Figure 1B). We then identified and conducted analyses on the frequency bands showing higher coherence values than other bands in both spectrums obtained from the averaged coherence in the two categories.

In order to confirm that the high coherence detected by the above-mentioned processes was not generated by aliasing of the physiological confounds contained in the hemodynamic signals, and could not be attributed to measurement modality (e.g., fMRI), we investigated coherence spectrums with NIRS signals. By projecting vitamin tablets onto cortical surfaces from structural MR images, we identified ROIs where NIRS signals were obtained. Two ROIs were identified within brain regions constituting the fronto-parietal system, and one ROI was located within the brain region forming the default mode system. In order to compare the results with those obtained using fMRI, we included in our analyses only the three NIRS channels located within brain regions included in the abovementioned resting-state functional connectivity systems. We then calculated the coherence between two ROIs in the fronto-parietal system and among ROIs between the fronto-parietal and default mode systems as counterparts of coherence for intra- and inter-systems.

\section{GRAPH METRICS FOR SEGREGATION AND INTEGRATION}

Network structures can be indices of the degree of information segregation and integration. For example, communities are sets of nodes in a network with dense interconnections. Their existence in a network enhances both communications within individual communities and independences between different communities-that is, segregation. Metrics that quantify how clearly communities in a network segregate can be used to investigate the degree of information segregation. By contrast, network structures such as hubs, which promote efficient communication among different nodes, can be indices of information integration. In another words, we can quantify the degree of information integration using graph metrics that measure how efficiently the nodes in a network communicate with each other. In the present study, we selected modularity as a measure of information segregation, and global efficiency as a measure of information integration.

\section{Frequency-specific network construction in individual data sets}

To define frequency-specific networks, we obtained adjacency matrices $A$ by applying the sparsity thresholds $S$, ranging from 0.05 to 0.25 in 0.05 increments, to the coherence matrices 
Table 1 | List of coordinations of ROIs.

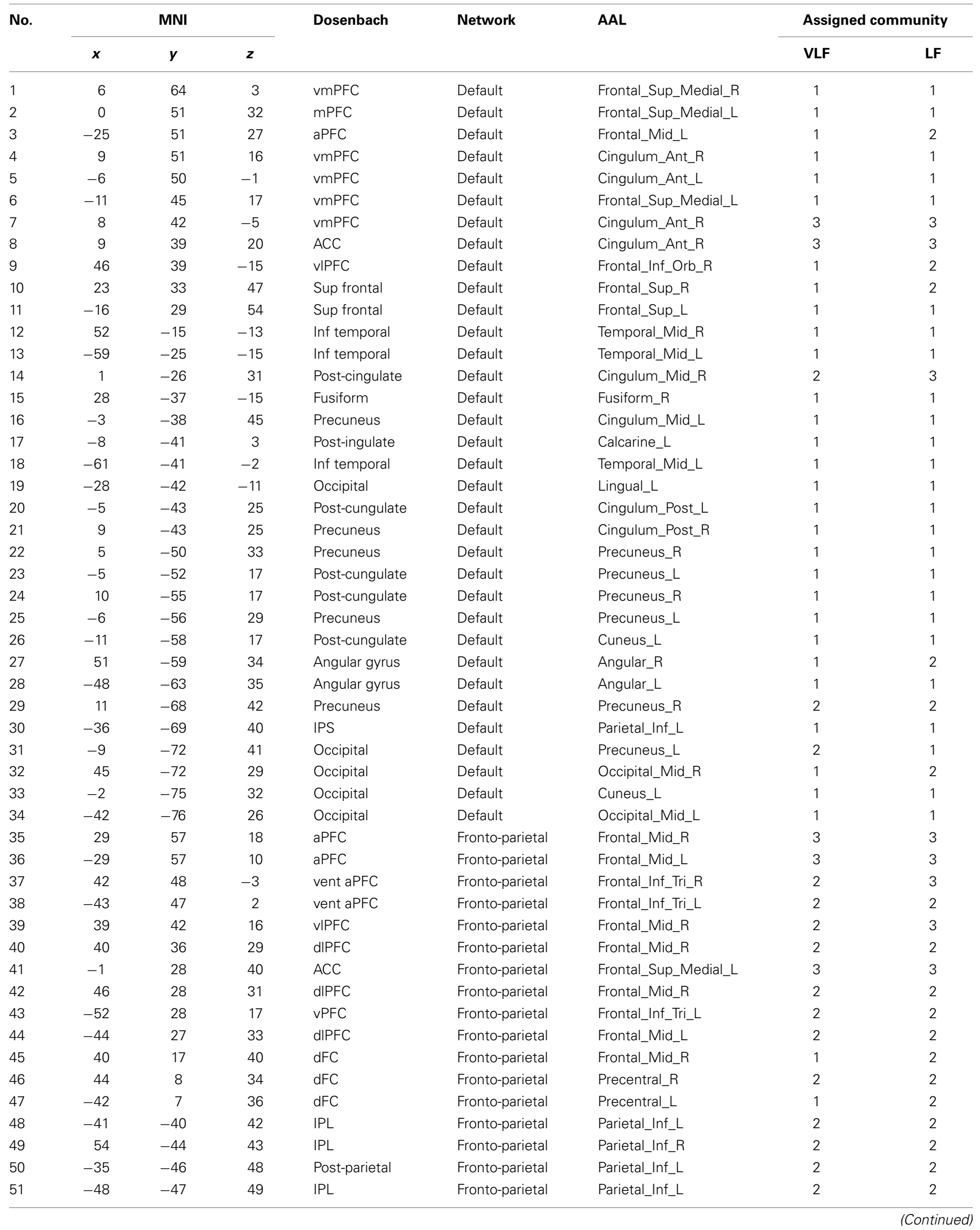


Table 1 | Continued

\begin{tabular}{|c|c|c|c|c|c|c|c|c|}
\hline No. & \multicolumn{3}{|c|}{ MNI } & Dosenbach & Network & AAL & \multicolumn{2}{|c|}{ Assigned community } \\
\hline 52 & -53 & -50 & 39 & IPL & Fronto-parietal & Parietal_Inf_L & 2 & 2 \\
\hline 53 & 44 & -52 & 47 & IPL & Fronto-parietal & Parietal_Inf_R & 2 & 2 \\
\hline 54 & -32 & -58 & 46 & IPS & Fronto-parietal & Parietal_Inf_L & 2 & 2 \\
\hline 55 & 32 & -59 & 41 & IPS & fronto-parietal & Angular_R & 2 & 2 \\
\hline 57 & 34 & 32 & 7 & VPFC & Cingulo-opercular & Frontal_Inf_Tri_R & 2 & 3 \\
\hline 58 & -2 & 30 & 27 & $\mathrm{ACC}$ & Cingulo-opercular & Cingulum_Mid_L & 3 & 3 \\
\hline 59 & 51 & 23 & 8 & $\mathrm{vFC}$ & Cingulo-opercular & Frontal_Inf_Tri_R & 1 & 2 \\
\hline 60 & 38 & 21 & -1 & Ant insula & Cingulo-opercular & Insula_R & 3 & 3 \\
\hline 61 & 9 & 20 & 34 & $\mathrm{dACC}$ & Cingulo-opercular & Cingulum_Mid_R & 3 & 3 \\
\hline 66 & -20 & 6 & 7 & Basal ganglia & Cingulo-opercular & Putamen_L & 3 & 3 \\
\hline 67 & 14 & 6 & 7 & Basal ganglia & Cingulo-opercular & Caudate_R & 3 & 3 \\
\hline 68 & -48 & 6 & 1 & $\mathrm{vFC}$ & Cingulo-opercular & Insula_L & 3 & 3 \\
\hline 69 & 37 & -2 & -3 & Mid insula & Cingulo-opercular & Putamen_R & 3 & 3 \\
\hline 70 & -12 & -3 & 13 & Thalamus & Cingulo-opercular & Caudate_L & 3 & 3 \\
\hline 71 & -12 & -12 & 6 & Thalamus & Cingulo-opercular & Thalamus_L & 3 & 3 \\
\hline 72 & 11 & -12 & 6 & Thalamus & Cingulo-opercular & Thalamus_R & 3 & 3 \\
\hline 73 & 32 & -12 & 2 & Mid insula & Cingulo-opercular & Putamen_R & 3 & 3 \\
\hline 74 & -30 & -14 & 1 & Mid insula & Cingulo-opercular & Putamen_L & 3 & 3 \\
\hline 82 & 43 & -43 & 8 & Temporal & Cingulo-opercular & Temporal_Mid_R & 1 & 1 \\
\hline 83 & -55 & -44 & 30 & Parietal & Cingulo-opercular & SupraMarginal_L & 2 & 2 \\
\hline 84 & 42 & -46 & 21 & Sup temporal & Cingulo-opercular & Angular_R & 1 & 1 \\
\hline 85 & -41 & -47 & 29 & Angular gyrus & Cingulo-opercular & Angular_L & 1 & 2 \\
\hline 86 & -59 & -47 & 11 & Temporal & Cingulo-opercular & Temporal_Mid_L & 1 & 1 \\
\hline 87 & -52 & -63 & 15 & TPJ & Cingulo-opercular & Temporal_Mid_L & 1 & 1 \\
\hline
\end{tabular}

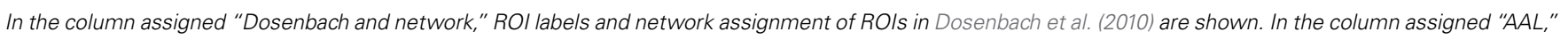

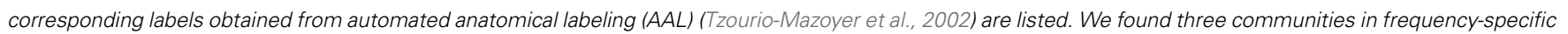

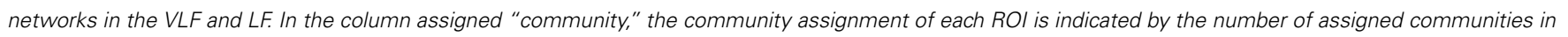
each frequency band (VLF and LF).

corresponding to the frequency bands showing higher coherence values than others (see above). Coherence matrices were calculated by averaging the coherence values within the frequency bands, which are a part of the matrices shown in Figure 1A. Sparsity $S$ was defined as the number of edges in a graph, divided by the maximum possible number of edges, and was used to measure the threshold (Latora and Marchiori, 2001; Achard and Bullmore, 2007):

$$
S=\frac{1}{n(n-1)} \sum_{i \in N} d_{i}
$$

where $n$ is the number of nodes in a graph $N, i$ is a node in graph $N$, and $d_{i}$ is the number of edges connected to the node $i$. Unlike a threshold using the value quantifying the strength of functional connectivity, $S$ can control the number of edges in the network 

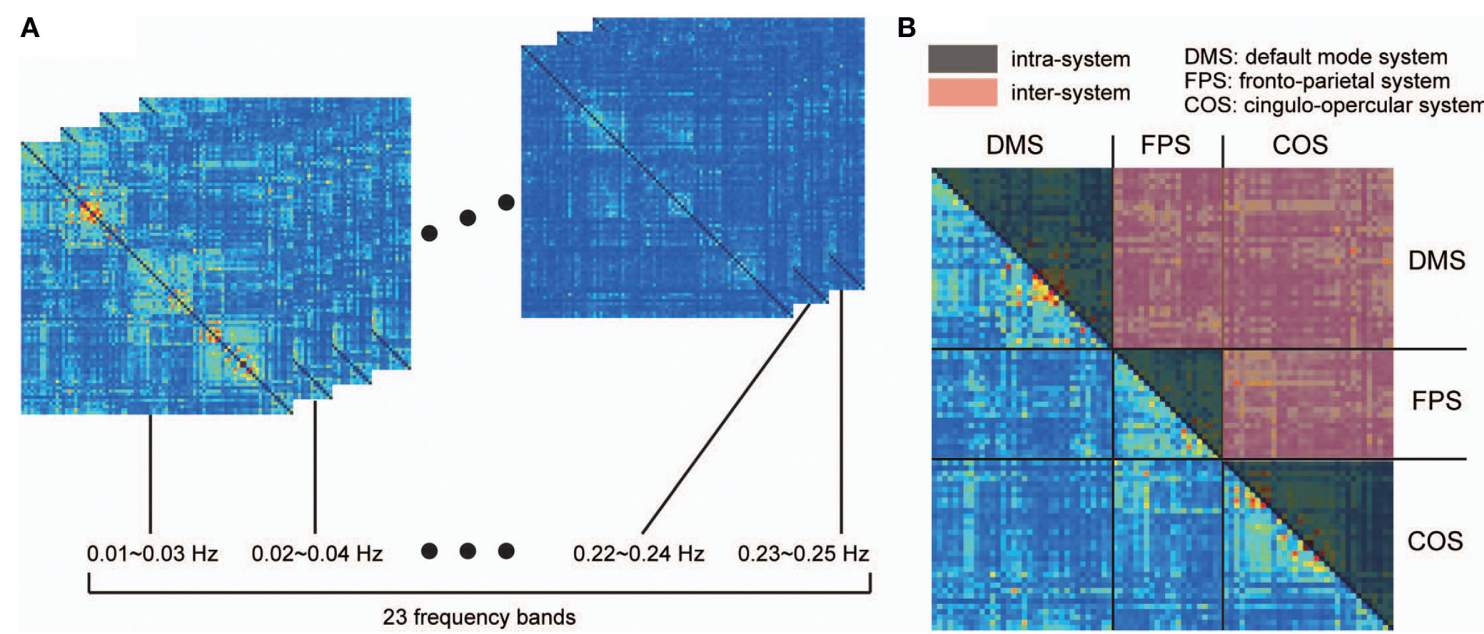

FIGURE 1 | Procedure for detecting frequency specificity of functional connectivity. Coherence was estimated in all pairs of ROls between 0.01 and $0.25 \mathrm{~Hz}$, and averaged within narrow, 50\%-overlapping frequency bands that had a band width of $0.02 \mathrm{~Hz}$. As a result, we obtained coherence matrices from 23 frequency bands (A). (B) Considering that frequency specificity is different between ROls in the same functional system and ROls of two distinct functional systems, coherence values were divided into two categories: coherence values within the same functional system (intra-system), and coherence values between different functional systems (inter-system). The colored parts of the matrix in (B) correspond to the coherence values of the intra-system (black) and inter-system (red). To investigate frequency specificity in these categories, the coherence values were further averaged within each category in each coherence matrix. between different conditions. Because many network metrics are affected by the number of edges in a graph, using $S$ allows us to attribute the different results of graph measures to differences in patterns of network connections. When one $S$ is selected, the corresponding thresholding value of the strength of functional connectivity is determined. Therefore, the range of $S$ should be determined so that the corresponding thresholding value of the measure of functional connectivity is significantly higher than 0 . To choose the lower bound of $S$, we calculated the null distributions of 10,000 coherence values by repeating calculations of shuffled signals obtained by a bootstraping method for each ROI pair. By ensuring the statistical significance $(p<0.05)$ of the coherence values corresponding to the sparsity thresholds for all participant data sets, we selected the lower bound of the sparsity thresholds as $S=0.25$.

\section{Modularity}

Modularity Q (Newman, 2004, 2006) indicates the extent to which the network can be subdivided into non-overlapping communities. For a set of non-overlapping communities $M, Q$ is defined as:

$$
Q=\sum_{u \in M}\left[e_{u u}-\left(\sum_{v \in M} e_{u v}\right)^{2}\right]
$$

where by $u$ and $v$ are communities, and $e_{u v}$ is the fraction of all links that connect nodes in $u$ with nodes in $v$.

\section{Global efficiency}

Global efficiency E (Latora and Marchiori, 2001) is an indicator of the global efficiency of parallel information transfer in the network and is defined as follows:

$$
E=\frac{1}{n} \sum_{i \in N} \frac{\sum_{j \in N, j \neq i} d_{i j}^{-1}}{n-1}
$$

In this case, $n$ is the number of nodes in the network, $N$ is the set of all nodes, and $d_{i j}$ is the shortest path length between node $i$ and node $j$.

All of these metrics were computed for each sparsity threshold in each individual data set using the Brain Connectivity Toolbox (Rubinov and Sporns, 2010).

\section{Statistical comparisons}

For each threshold level, we conducted a two-tailed $t$-test with subjects (random effects analysis) against the null hypothesis, defined as no significant group-level difference between graph metrics calculated in two frequency bands that showed higher coherence values than other bands. Because we used 5 different sparsity thresholds and 2 graph theoretical metrics, correction was conducted with considering 10 multiple comparisons. The false discovery rate (FDR) method was used to correct for multiple comparisons, and significant differences were detected at $p<0.05$ after FDR correction (Benjamini and Yekutieli, 2001).

\section{IDENTIFICATION OF FREOUENCY-SPECIFIC NETWORK STRUCTURES FOR SEGREGATION AND INTEGRATION}

Graph metrics can reveal network attributes for information segregation and integration; however, it is not clear which network structures realize these informational properties. Brain networks possess characteristic structures that play key roles in segregation and integration; yet it remains unclear whether these structures can be consistently found in frequency-specific networks. To tackle this issue, we estimated group-level network structures. 
For each group-level frequency-specific network, we identified communities as structures for information segregation, and hubs and rich-clubs (higher-order structures of hubs) as structures for information integration.

\section{Group-level network construction}

Network connection patterns have inter-individual variability. In order to investigate consistencies in structure at the group level, we constructed a network-level adjacency matrix $A^{g}$ from the individual-level adjacency matrices $A$ of all individual data sets. $A$ is a binary matrix, defined as $A_{i j}=1$ when there is functional connectivity between node $i$ and $j$, otherwise it is defined as $A_{i j}=$ 0 . Adjacency matrix $A$ was calculated for each frequency band in each participant's dataset. We generated a matrix representing the consistency of functional connectivity across all participants by averaging $A$ in each frequency band. We refer to this here as the consistent edge matrix $C e\left(0 \leqq C e_{i j} \leqq 1\right)$. Then, by applying sparsity thresholds on $C e$, we obtained $A^{g}$ in frequency bands where the coherence showed higher values than other frequency bands in both spectrums obtained from the averaged coherence in the two categories. Although the same sparsity thresholds $S$ between 0.05 and 0.25 for the 0.05 increments were used in producing $A$ and $A^{g}$, we finally selected $S$ s generating connected $A^{g}$, which are graphs in which there is at least one direct or indirect pathway among all nodes, for all frequency bands showing higher coherence values than others.

\section{Community detection}

We then examined group-level community structures in grouplevel networks obtained in different frequency bands. As community structures have between-participant variability, group-level community structures were not identified with the consistent edge matrix $C e$, but rather were detected with the consistent assignment matrix $\mathrm{Ca}$ (van den Heuvel et al., 2008a; Fornito et al., 2012). This matrix was constructed as follows. First, community detection was conducted on the adjacency matrix $A$ of each participant. Individual-level consistency of community assignment was expressed in a matrix $I C a$ in which the element $I C a_{i j}$ is 1 if the ROIs $i$ and $j$ are assigned in the same community. Then, ICas were averaged across participants to produce $C a$ in which the element $C a_{i j}$ represents the incidence of two ROIs being assigned to an identical community within the group $\left(0 \leqq C a_{i j} \leqq 1\right)$. Finally, by applying the community detection algorithm on Cas, we estimated group-level community structures in all frequency bands with higher coherence values than others. Several algorithms have been developed to identify optimal communities in a network. We compared the effectiveness of three community detection algorithms (Newman, 2006; Blondel et al., 2008; Sun et al., 2009) implemented in the Brain Connectivity Toolbox to estimate community structures. The Louvein method algorithm (MATLAB function in Brain Connectivity Toolbox, modularity_louvain_und.m) was selected for detecting communities as it returned the highest modularity.

Once communities were detected in each frequency-specific network, we assessed the similarity of the brain regions, formed by using normalized mutual information NMI, as proposed by Kuncheva and Hadjitodorov (2004), to quantify the similarity of different community assignments on the same node set (Meunier et al., 2009). NMI can quantify the accuracy with which one assignment of a given node set predicts the other, and it is defined as:

$$
N M I=\frac{-2 \sum_{i=1}^{c_{1}} \sum_{j=1}^{c_{1}} N_{i j} \log \left(\frac{N_{i j} N}{N_{i} N_{j}}\right)}{\sum_{i=1}^{c_{1}} N_{i} \log \left(\frac{N_{i}}{N}\right)+\sum_{j=1}^{c_{2}} N_{j} \log \left(\frac{N_{j}}{N}\right)} .
$$

where $C_{1}$ and $C_{2}$ are the number of detected communities for each assignment, $N$ is the number of nodes in the network, $N_{i}$ and $\mathrm{N}_{j}$ are the numbers of nodes assigned in $i$-th and $j$-th communities, and $N_{i j}$ is the number of nodes commonly assigned in both $N_{i}$ and $N_{j}$ in different partitions. The same assignments give $N M I=1$, while $N M I$ is 0 among totally independent assignments.

Statistical significance for the similarity between assignments of VLF and of LF was tested by generating 10,000 pairs of community assignments in randomized networks of $A^{g}$ s. This produced a null distribution consisting of the 10,000 NMIs. In addition, we calculated the NMI between the frequency-specific networks and community assignment reported in Dosenbach et al. (2010). The adjacency matrix in which the community assignment was calculated in Dosenbach et al. (2010) was not reported; therefore, we obtained the null distribution of the community assignment by generating 10,000 random networks in addition to 10,000 randomized $A^{g} \mathrm{~S}$, in order to test the statistical significance of the NMIs.

\section{Hub detection}

We detected hub regions in all group-level networks obtained in different frequency bands, in order to assess whether topological differences were reflected in different hub alignments between these networks. In order to identify hub regions, we measured two graph theoretical metrics for each node: the nodal degree and the eigenvector centrality (Lohmann et al., 2010). The nodal degree was calculated in group-level adjacency matrices $A^{g}$ s, whereas the eigenvector centrality was computed in consistent edge matrices Ces without applying a threshold. In the current study, we defined hubs as nodes in which both the nodal degree and the eigenvector centrality were at least one standard deviation above the network mean. The nodal degree and eigenvector centrality measure different aspects of nodes in the network; the nodal degree simply counts the number of edges connecting a node, whereas the eigenvector centrality estimates how a node can affect others connecting with it. As we aimed to identify hub regions with highly connected and highly central nodes in a network, we used both criteria to define them.

\section{Rich-club detection}

A study of human anatomical connectivity has shown that structural brain hubs are not independent of each other, but rather form a rich club. This is characterized by a tendency for high-degree nodes to be more densely anatomically connected among themselves than nodes of a lower degree (van den Heuvel and Sporns, 2011). The appearance of a rich club in human 
anatomical networks suggests that these regions, identified as structural brain hubs, perform some collaborative functions like information integration. This raises a question of whether hubs identified in FCNs also organize the rich-club.

By denoting the number of nodes with a higher degree than $k$ as $N_{k}$, and designating the edges within the sub-network that consist of these nodes as $E_{k}, k$-density $\Phi(k)$ is defined as follows:

$$
\varphi(k)=\frac{2 E_{k}}{N_{k}\left(N_{k}-1\right)} .
$$

The denominator represents the maximal number of edges within the sub-network. Several graphs including a random network, in which nodes are interconnected by chance, show that $\Phi(k)$ grows with $k$. Therefore, if there is a tendency for hubs to be more inter-connected than nodes of a lower degree, $\Phi(k)$ increases with $k$ at a higher rate than those expected out of random networks (Colizza et al., 2006; McAuley et al., 2007)—that is, $\Phi(k)$ is informative when this coefficient is normalized by the expected one. Therefore, we identified a range of $k$-values expressing this characteristic as follows, and subsequently refer to such phenomena as a "rich-club regime" (van den Heuvel and Sporns, 2011). First, we calculated $\Phi(k)$ for all $A^{g}$ s. Then, we constructed 1000 randomized networks for each of the $A^{g}$ s using the Brain Connectivity Toolbox, and computed 1000 coefficients in these networks $\Phi_{\text {randomized }}(k)$. We defined the range of $k$ where $\Phi(k)$ was significantly higher than the values calculated in the randomized networks. To evaluate statistical significance, we compared $\Phi(k)$ with the distribution consisting of $1000 \Phi_{\text {randomized }}(k) \mathrm{s}$ and identified the range where $\Phi(k)$ s were consistently included within the upper $1 \%$ of the distribution. When we detected more than two ranges satisfying this condition, we defined a richclub regime as the highest range. Finally, normalized rich-club coefficients $\Phi_{\text {normalized }}(k)$ were calculated by dividing $\Phi(k)$ with $\Phi_{\text {meanrand }}(k)$, which represents the mean of $1000 \Phi_{\text {randomized }}(k)$ s.

\section{Characterizing the functional role of hubs}

The functional role of a hub in a network can be determined via the relationship with communities. For example, hubs with connections that are mostly within a single community (intracommunity connections) facilitate integration within that community, whereas those with multiple connections to different communities (inter-community connections) promote integration among communities. The participation coefficient $P$ is a graph theoretical metric that expresses the distribution of intravs. inter-community connections (Guimera and Amaral, 2005). The $P$-value of an individual node, $P_{i}$, is defined as follows:

$$
P_{i}=1-\sum_{s=1}^{N_{M}}\left(\frac{\kappa_{i s}}{k_{i}}\right)^{2},
$$

where $\mathrm{N}_{M}$ is the number of identified communities in a network, $k_{i}$ is the degree of the node, and $\kappa_{i s}$ is the number of edges linking the node and other nodes within a community expressed by the subscript s. Guimera and Amaral (2005) have shown that, by using this metric, hubs can be naturally divided into three different roles: provincial hubs $(P \leq 0.30)$, connector hubs
$(0.30<P \leq 0.75)$, and kinless hubs $(P>0.75)$. The application of this metric in brain networks has successfully characterized the functional roles of hubs in cats, macaques, and humans (Sporns et al., 2007; Hagmann et al., 2008; Meunier et al., 2009; Power et al., 2013). In the current study, we used this metric to characterize the functional role of detected hubs in frequency-specific FCNs.

\section{RESULTS}

\section{FREOUENCY-DEPENDENCY OF FUNCTIONAL CONNECTIVITY}

Group-averaged coherence values were calculated and averaged within the following two categories of ROI pairs: ROIs within the same functional system (intra-system) and ROIs belonging to different functional systems (inter-system; Dosenbach et al., 2010; Figure 1B). The highest value of the averaged coherence in the intra-system was observed in the lowest frequency band; hereafter we refer to this frequency band $(0.01-0.03 \mathrm{~Hz})$ as VLF (very low frequency) represented by its center frequency $(0.02 \mathrm{~Hz})$ in Figure 2. There was one other frequency band in which the coherence values were higher than others; we call this frequency band $(0.07-0.09 \mathrm{~Hz}$ ) as the LF (low frequency band) indicated as $0.08 \mathrm{~Hz}$ in Figure 2. In both frequency bands, we found that the averaged coherence value obtained in the inter-system was also higher than other frequency bands. The result did not depend on the width of frequency bands where coherence values were averaged (Figure S1). In order to ensure that the observed frequency characteristics were reproducible in data sets obtained at other institutions, we estimated the abovementioned coherence spectrum in a public resting state fMRI data set with 96 participants from the 1000 Functional Connectome Project (http://fcon_1000.projects.nitrc.org/index.html). Results confirmed that coherence values in the VLF and LF were larger than those in other frequency bands (Figure S2). Furthermore, to confirm that this frequency specificity was not due to aliasing of physiological noise contained in higher frequency regions, we investigated the coherence spectrum of a simultaneously obtained NIRS data set. NIRS signals were measured with a sufficiently higher sampling rate $(10 \mathrm{~Hz})$ to characterize hemoglobin signals including respiratory and cardiac pulsations, which were observed as separate peaks in the power spectrum. We confirmed that although there were peaks corresponding to typical respiratory and cardiac pulsations around 0.3 and $1 \mathrm{~Hz}$ in the coherence spectrum, VLF and LF were still signature frequency bands where the coherence values were higher than other frequency bands within the $0.01-0.10 \mathrm{~Hz}$ band (Figure 3). Collectively, these results demonstrate that there are two frequency components that strongly contribute to resting state functional connectivity within the frequency band $(0.01-0.10 \mathrm{~Hz})$, where functional connectivity has been estimated in many studies. Thus, we focused and conducted analyses on these two frequency bands.

\section{GRAPH METRICS FOR SEGREGATION AND INTEGRATION}

To construct frequency-specific networks identified in the VLF and LF bands for each individual data set $\left(I_{\mathrm{VLF}}\right.$ and $\left.I_{\mathrm{LF}}\right)$, we applied five sparsity thresholds to two band-averaged coherence matrices, and calculated graph theoretical metrics using 


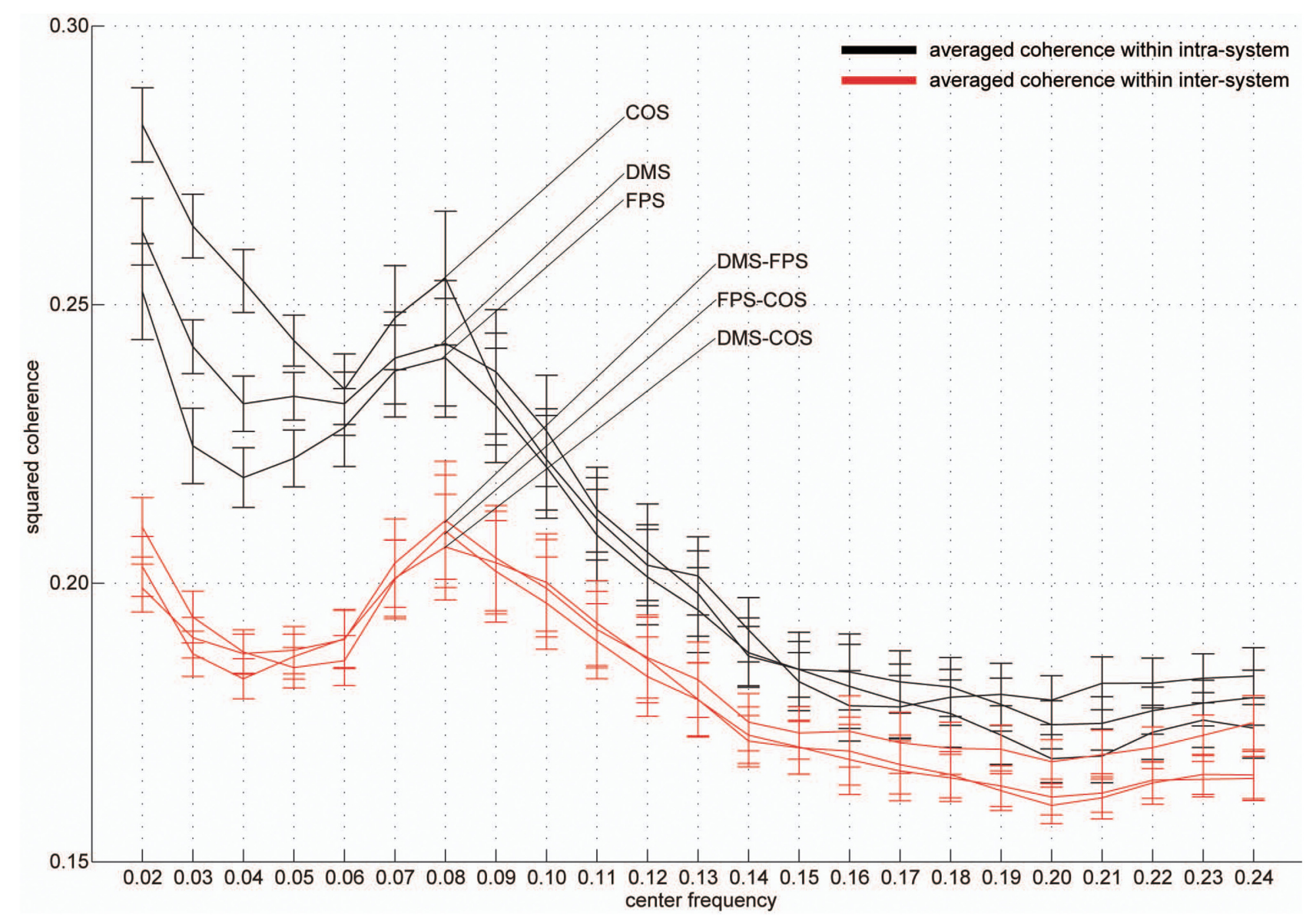

FIGURE 2 | Frequency-specificity of functional connectivity. Averaged coherence values in two categories are shown. Black curves represent coherence values averaged within three functional systems and red curves indicate values calculated in the inter-system groups (Figure 1). Error bars show the standard errors. The x-axis represents the center frequencies of the frequency bands, where coherence values were averaged. For all curves, coherence within $0.01-0.03 \mathrm{~Hz}$ (very low frequency, [VLF]) and $0.07-0.09 \mathrm{~Hz}$ (low frequency, [LF]) were higher than those of other frequency bands. the adjacency matrices $A$. We then conducted two-tailed $t$-tests against the null-hypothesis that there were no group-level differences between $I_{\mathrm{VLF}}$ and $I_{\mathrm{LF}}$ in the calculated graph metrics. For all sparsity levels, the modularity, which is a graph metric of segregation, obtained in the $I_{\mathrm{VLF}}$ was significantly higher than in the $I_{\mathrm{LF}}(p<0.05$, corrected; Figure 4A); this demonstrated that the $I_{\mathrm{VLF}}$ had a significantly higher capacity for information segregation than the $I_{\mathrm{LF}}$. By contrast, no significant differences were found in global efficiency (a measure of information integration; Figure 4B).

\section{FREQUENCY-SPECIFIC NETWORK STRUCTURES FOR SEGREGATION}

We thresholded the consistent edge matrix $C e$ by applying the sparsity thresholds $S$, ranging from 0.05 to 0.25 in 0.05 increments, to obtain group-level frequency-specific networks, $A^{g}$, in both the VLF and LF ranges $\left(A_{\mathrm{VLF}}^{g}\right.$ and $\left.A_{\mathrm{LF}}^{g}\right)$. It was only when we applied $S=0.25$ as a threshold that both $A_{\mathrm{VLF}}^{g}$ and $A_{\mathrm{LF}}^{g}$ became a connected graph. Therefore, we conducted the following analyses using this sparsity threshold.

By detecting consistent community structures across participants for both networks (Figures 5A,B), we found three communities in both $A_{\mathrm{VLF}}^{g}$ and $A_{\mathrm{LF}}^{g}$. The correspondence between each region and the assigned community is shown in Table 1. We calculated NMI to quantify the similarity of community assignments and found that detected communities in $A_{\mathrm{VLF}}^{g}$ and $A_{\mathrm{LF}}^{g}$ were significantly similar to each other $(N M I=0.59 ; p<$ $0.0001)$. Comparing the NMI between the community assignments identified in the frequency-specific networks and those reported by Dosenbach et al. (2010) revealed that assignments in both $A^{g}$ VLF and $A^{g}$ LF showed significant similarity with the reported assignment $\left(0.33\right.$ for $A^{g}{ }_{\mathrm{VLF}}[p<0.0001]$; 0.32 for $A^{g} \mathrm{LF}$ $[p<0.0001])$. This supports that we could consistently find three communities analogous to DMS, FPS, and COS in both $A^{g}$ VLF and $A^{g} \mathrm{LF}$. (community assigned Nos. 1, 2 and 3 in Table 1). These findings suggest that, although these communities were more strongly segregated in $A^{g} \mathrm{VLF}$ than $A^{g} \mathrm{LF}$, both consisted of three communities corresponding to functional systems.

\section{FREOUENCY-SPECIFIC NETWORK STRUCTURES FOR INTEGRATION}

In order to identify hub regions in frequency-specific networks, we calculated nodal degrees and eigenvectors for $A_{\mathrm{VLF}}^{g}$ and $A_{\mathrm{LF}}^{g}$ 


\section{A}

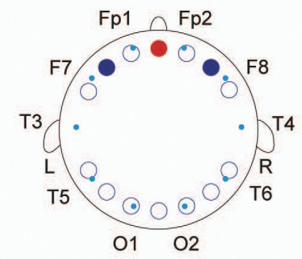

- Reference points

- Measured regions by NIRS

A region of default mode

system (median prefrontal cortex)

Regions of fronto-parietal

- system (bilateral anterior prefrontal cortices)
B

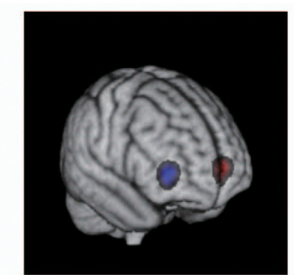

C

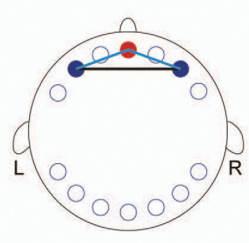

Intra-system (within

fronto-parietal system)

Inter-system (between

- default mode and frontoparietal systems)
D

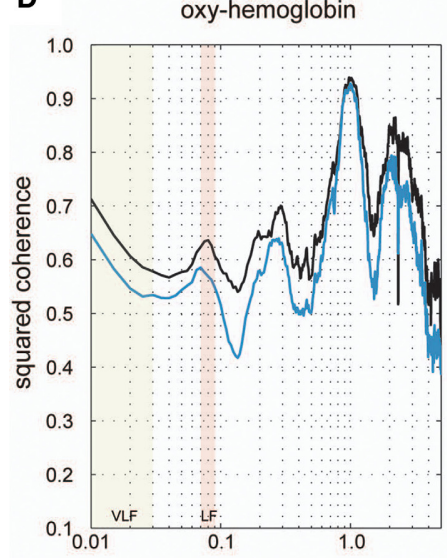

E

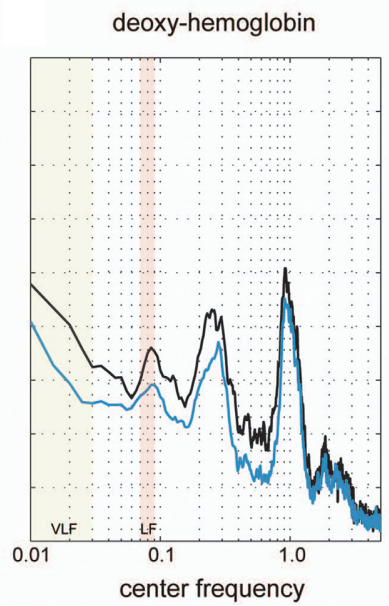

$\mathbf{F}$
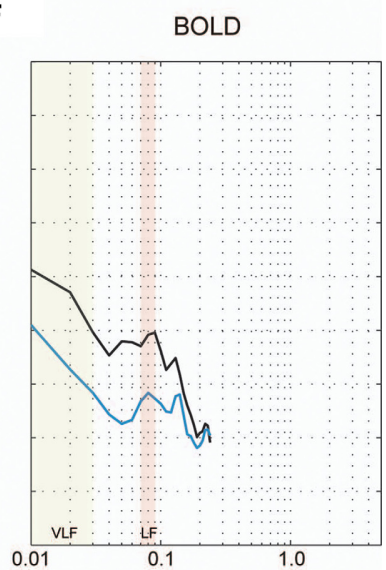

FIGURE 3 | Coherence spectrum estimated using simultaneously obtained NIRS data. (A) We obtained NIRS signals at 14 cortical regions indicated as blue rings. Cyan dots represent standard reference points used in locating channels of electroencephalography on the scalp. In a previous study, we identified 14 cortical regions, where NIRS signals were obtained, in MNI space to determine ROIs corresponding to each NIRS measurement region for each individual. For detailed methods for the identification and $\mathrm{MNI}$ coordinates, please refer to Sasai et al. (2012). As a result, we found one cortical region (medial prefrontal cortex [mPFC]) included in the default mode system (red filled circle) and two bilateral cortical regions (left and right anterior prefrontal cortices [laPFC and raPFC]) contained in the fronto-parietal system (blue filled circles). (B) Voxels corresponding to measured regions by NIRS are shown. Colors are the same as those defined in (A). (C) We calculated the coherence between laPFC and raPFC to investigate the intra-system coherence spectrum (fronto-parietal system), and also estimated the coherence between MPFC and laPFC, and between MPFC and raPFC, to examine the inter-system coherence spectrum (default-mode and fronto-parietal systems). A black line indicates an intra-system pair of ROls, whereas cyan lines represent inter-system pairs. (D,E) Coherence spectrums of two NIRS signals (oxygenated [oxy-] hemoglobin and deoxygenated [deoxy-] hemoglobin) with two clear peaks corresponding to typical frequency bands of respiratory fluctuation around $0.3 \mathrm{~Hz}$ and cardiac pulsations around $1 \mathrm{~Hz}$. High coherences in VLF and LF could still be observed in the spectrum, supporting the idea that higher coherences in these bands are not due to aliasing. (F) Coherence spectrum obtained using fMRI signals extracted from ROIs corresponding to NIRS measurement regions (as shown in B). We confirmed the high coherence values in VLF and LF in this spectrum, supporting the notion that characteristics of the coherence spectrum cannot be attributed to differences in ROI locations between our current and previous studies.
(Figure 6). In both metrics, we identified high degree and high centrality nodes with metrics greater than the network mean plus one standard deviation (yellow bars in Figures 6A-D). Hub regions were then defined as ROIs detected as both high degree and high centrality nodes (Table 2). While seven ROIs were identified as hubs for both $A_{\mathrm{VLF}}^{g}$ and $A_{\mathrm{LF}}^{g}$, all hubs except the one for the left dorsal anterior precuneus cortex (ldaPrCC, [AAL: Cingulum_Mid_L]) were different between $A_{\mathrm{VLF}}^{g}$ and $A^{g} \mathrm{LF}$. Frequency-specific hubs in $A_{\mathrm{VLF}}^{g}$ were detected in the left superior medial frontal cortex, left supplementary motor area, left middle, and right anterior cingulate cortices, all of which were regions assigned in one community (No. 3 in Table 2). This consisted of similar regions to the COS. Frequency-specific hubs in $A_{\mathrm{LF}}^{g}$ were detected in the left cuneus, right precuneus, and right thalamus. Contrary to $A_{\mathrm{VLF}}^{g}$, the frequency-specific hubs in $A_{\mathrm{LF}}^{g}$ were distributed over all communities.

Figures 6E,F show the rich-club coefficient curves obtained in both $A^{g}$ VLF and $A^{g}$ LF. We found a range of $k$-values showing 

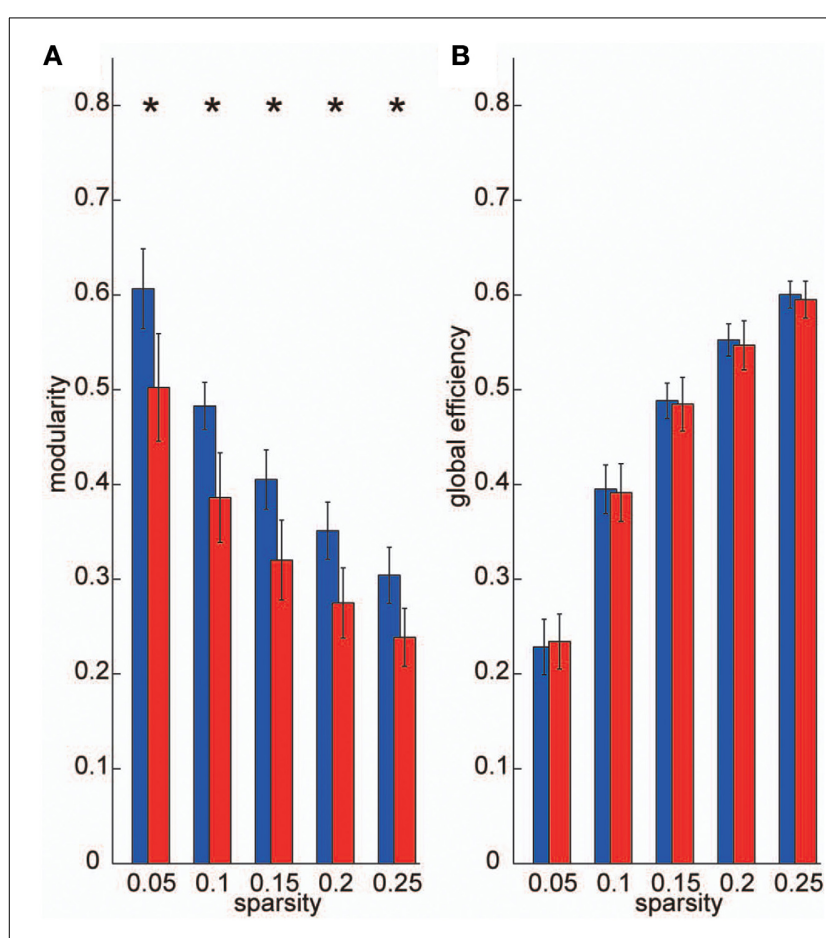

FIGURE 4 | Graph metrics. We calculated the following two graph metrics in two frequency-specific networks (VLF and LF) estimated in each individual data set: (A) modularity, and (B) global efficiency. Blue bars represent the mean of each graph metric obtained, which was computed in networks estimated in the VLF. Red bars indicate the mean graph metric in the LF. In the current study, we selected sparsity of the brain networks (number of existing edges over the maximum possible number of edges) as threshold measurements. Because different threshold values might affect these graph metrics, we examined the between-group differences in these parameters over a wide range of threshold levels (0.05-0.25). Asterisks indicate statistically significant differences between the metrics obtained in the VLFN and the LFN, tested by two-sampled $t$-tests ( $p<0.05$, false discovery rate-corrected).

significantly higher rich-club coefficients than those calculated in randomized topologies for both $A^{g} \mathrm{VLF}$ and $A^{g} \mathrm{LF}$. The richclub regime in $A^{g}$ VLF was $29 \leqq k \leqq 31$ vs. $10 \leqq k \leqq 39$ in $A^{g}$ LF. In the rich-club regime observed in each frequency band, we found a value of $k$ at which the rich-club organization in each frequency-specific network was formed by a detected hub region $\left(k=30\right.$ for $A^{g}{ }_{\mathrm{VLF}}$ and $k=36$ for $\left.A^{g} \mathrm{LF}\right)$. This demonstrated that significantly dense interconnections exist among hubs in each frequency-specific network.

The functional roles of the identified hubs were estimated by calculating the participation coefficient $P$ in both $A^{g}$ VLF and $A^{g}{ }_{\mathrm{LF}}$ (Figures 6G,H). As $P$ cannot exceed 0.67 for networks consisting of three communities, we cannot observe kinless hubs (hubs with $P>0.75$; see Materials and Methods and Guimera and Amaral, 2005) in both frequency-specific networks. We found that all detected hubs in both $A^{g}$ VLF and $A^{g}$ LF were classified in connector hubs $(0.3 \leq P<0.75)$, indicating that hubs have a role in integration among the three detected communities in both $A^{g}$ VLF and $A^{g}$ LF.

Anatomical perspectives of hub regions in both networks are shown in Figure 7. Although several ROIs have functional connectivity with hub regions in each network, among the hubs there were dense interconnections or rich-club connections. This finding demonstrates that although there were no significant differences regarding global efficiency between the two frequencyspecific networks, their structures contributing to information integration consisted of distinct sets of functional brain hubs that formed distinct rich-club organizations.

Although hub regions in the VLF mainly contained areas of the anterior cingulate and superior medial frontal cortices, those in the LF consisted of the precuneus cortex and thalamus. In order to investigate whether this difference was specific to the relationship between VLF and LF, we identified hub regions within the typical frequency band used for studies of functional connectivity $(0.01-0.10 \mathrm{~Hz})$ and within three frequency bands (bandwidth $0.02 \mathrm{~Hz}$ ) located within $0.01-0.11 \mathrm{~Hz}$ without overlapping with the VLF and the LF $(0.03-0.05,0.05-0.07$, and $0.09-0.11 \mathrm{~Hz}$; Figure 7G). Within the $0.01-0.10 \mathrm{~Hz}$ band, we observed hub regions located in the anterior and posterior cingulate cortices and the thalamus. This supports the notion that network characteristics of integration in the VLF and LF coexist within the network obtained in the wide frequency band. We found that hub regions identified in the frequency bands higher than $0.05 \mathrm{~Hz}$ mainly and consistently included ROIs in the precuneus and thalamus, thus supporting the notion that hub regions in the LF reflect representative integration architecture at this frequency range. By contrast, the VLF was the only frequency band where hubs mainly consisted of the medial frontal regions. In the frequency band between 0.03 and $0.05 \mathrm{~Hz}$, which was located between the VLF and frequency ranges over $0.05 \mathrm{~Hz}$, hub regions were identified in both the medial frontal and parietal regions. The network topology of this frequency band might reflect functional characteristics of both networks estimated in the VLF and LF (Figure 7G).

\section{DISCUSSION}

This study investigated frequency specificity of a functional network architecture contributing to information segregation and integration. By calculating coherence among all pairs of ROIs, we found two frequency bands within the range $0.01-0.10 \mathrm{~Hz}$, VLF and LF, where coherence was higher than other frequency bands (Figure 2). Although graph theoretical metrics showed that the network estimated in the VLF had a higher degree of segregation than that in the LF (Figure 4A), no difference was found regarding indices of integration (Figure 4B). By contrast, both frequency-specific networks could be decomposed into a highly similar set of communities corresponding to three functional brain systems (the DMS, FPS, and COS; Figure 5). This indicates that, although networks in the VLF and LF consisted of the same community sets, these were more segregated in the VLF than in the LF. Furthermore, by identifying hub regions in each frequency-specific network, we observed that the hub regions differed in all frequency bands except for one region, the left dorsal anterior precuneus (Table 2); this supported the notion that there were at least two distinct sets of functional hubs depending on the timescale of brain activity. Collectively, our findings demonstrate spontaneous fMRI signal fluctuations in two different frequency bands organized into large-scale networks 


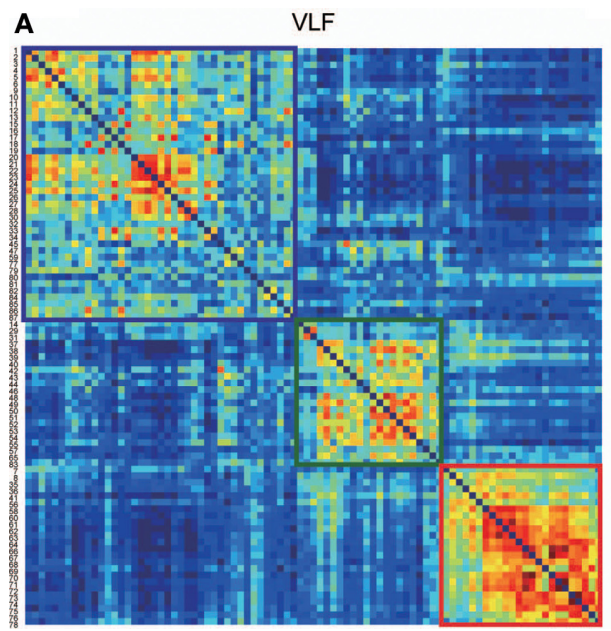

FIGURE 5 | Consistency of community detection between VLF and LF.

(A,B) Show consistent assignment matrices Ca obtained in the VLF (A) and LF (B). To emphasize the modular structures, both Ca were reordered by putting the ROls in the same module next to each other. Detecting

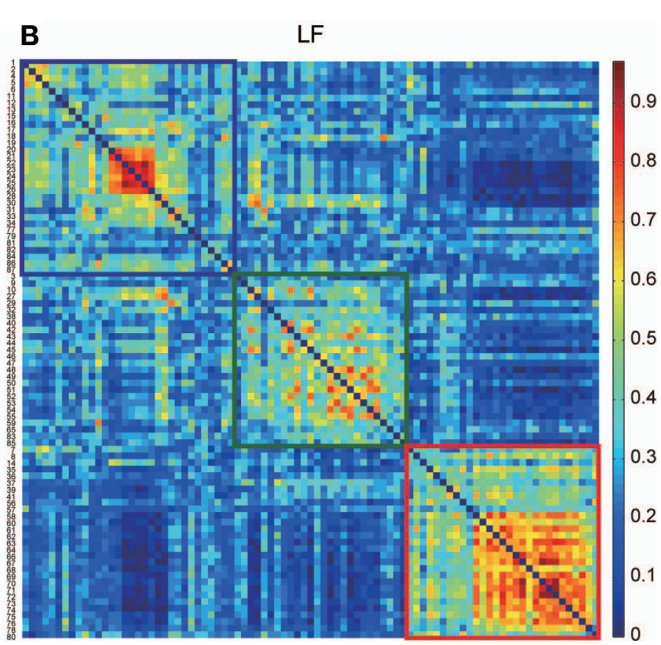

communities in both matrices revealed three in the VLF and LF indicated by squares. The color of each square corresponds to the assigned community detected in each frequency band: blue denotes community 1 , green community 2 , and red community 3 . with distinct topologies for information segregation and integration.

Spontaneous hemodynamic signals include not only fluctuations in spontaneous neural activity but also those generated by physiological signals such as respiratory and cardiac pulsations. Using NIRS has demonstrated that respiratory and cardiac pulsations have typical frequencies $(0.3$ and $1 \mathrm{~Hz})$, and dominate these frequency bands in the power spectrum (Obrig et al., 2000). Importantly, two additional frequency bands corresponding to the VLF and LF $(0.01-0.03 \mathrm{~Hz}$ and $0.06-0.08 \mathrm{~Hz})$ have been demonstrated, for which coherence values estimated by signal fluctuations of oxygenated hemoglobin concentration among some distant brain regions are higher than those values in other frequency regions within $0.01-0.10 \mathrm{~Hz}$ (Sasai et al., 2011). We obtained a similar result in the current study using both NIRS signals of oxygenated and deoxygenated hemoglobin concentration changes and simultaneously obtained fMRI data analyzed (Figure 3). These results demonstrate that high coherence in the VLF and LF is not due to aliasing of physiological signals in higher frequency regions, and high coherence in the VLF and LF can be consistently observed by different measurement modalities of hemodynamic signals - that is, fMRI and NIRS. Moreover, we conducted additional spectrum analyses on public resting state fMRI data sets and ensured that these characteristics of coherence spectra are universal features of resting state fMRI signals (Figure S1). These results support the hypothesis that high coherences in the VLF and LF of fMRI signals reflect coherent spontaneous neural activities.

In analyzing the brain from the view of graph theory, the minimal node is defined as each voxel in the MRI data set. Although there are studies constructing a voxel-based network of the brain (Eguíluz et al., 2005; Cecchi et al., 2007; van den Heuvel et al., 2008b; Buckner et al., 2009; Hayasaka and Laurienti, 2010), this method is computationally demanding. To reduce the volume of MRI data set, nodes are determined in a larger spatial scale as regions of interest (ROI). ROIs are generally determined by referring the boundary of brain regions that are activated by a specific category of tasks (Dosenbach et al., 2007, 2010; Deshpande et al., 2011; Power et al., 2011; Spreng et al., 2013) or defined as anatomically separate regions (Salvador et al., 2005a,b, 2007, 2008; Achard et al., 2006; Bassett et al., 2008; Hagmann et al., 2008). However, to obtain an accurate description of FCN, ROIs should be selected to represent underlying functional areas of the brain (Butts, 2009; Dosenbach et al., 2010). Therefore, we selected nodes as ROIs defined by a meta-analysis on several taskactivation studies in order to ensure reliability on the functional uniformity of voxels within ROIs (Dosenbach et al., 2010). The variability of selections of ROIs can cause inconsistency on results of the network topology. However, hubs and rich-clubs identified in the present study $(0.01-0.10 \mathrm{~Hz}$; see Table 2$)$ are located in the similar brain regions that have been repeatedly reported by previous studies using different ROI definitions (see Achard et al., 2006; van den Heuvel et al., 2008b; Buckner et al., 2009; Tomasi and Volkow, 2011a,b; Spreng et al., 2013; for hub; see van den Heuvel and Sporns, 2011 for rich-club). We observed a consistent community assignment with those reported in other studies using distinct ROI sets (Dosenbach et al., 2007; Power et al., 2011; Spreng et al., 2013) as well as a study using the same ROIs (Dosenbach et al., 2010). Thus, it is likely that all of our findings didn't depend on our selection of ROI.

There are several methods for detecting network structures such as communities and hubs; thus, different results might be obtained depending on the method selected. There are also several community detection algorithms for use within a network. However, the optimal algorithm can be selected using a measure of modularity, which has been used as an evaluation function (Newman, 2004, 2006). The algorithm used in the current study was selected because it provided the highest modularity among a given set. Once the optimal algorithm had been selected, the modularity was used as an index to quantify the degree of segregation 

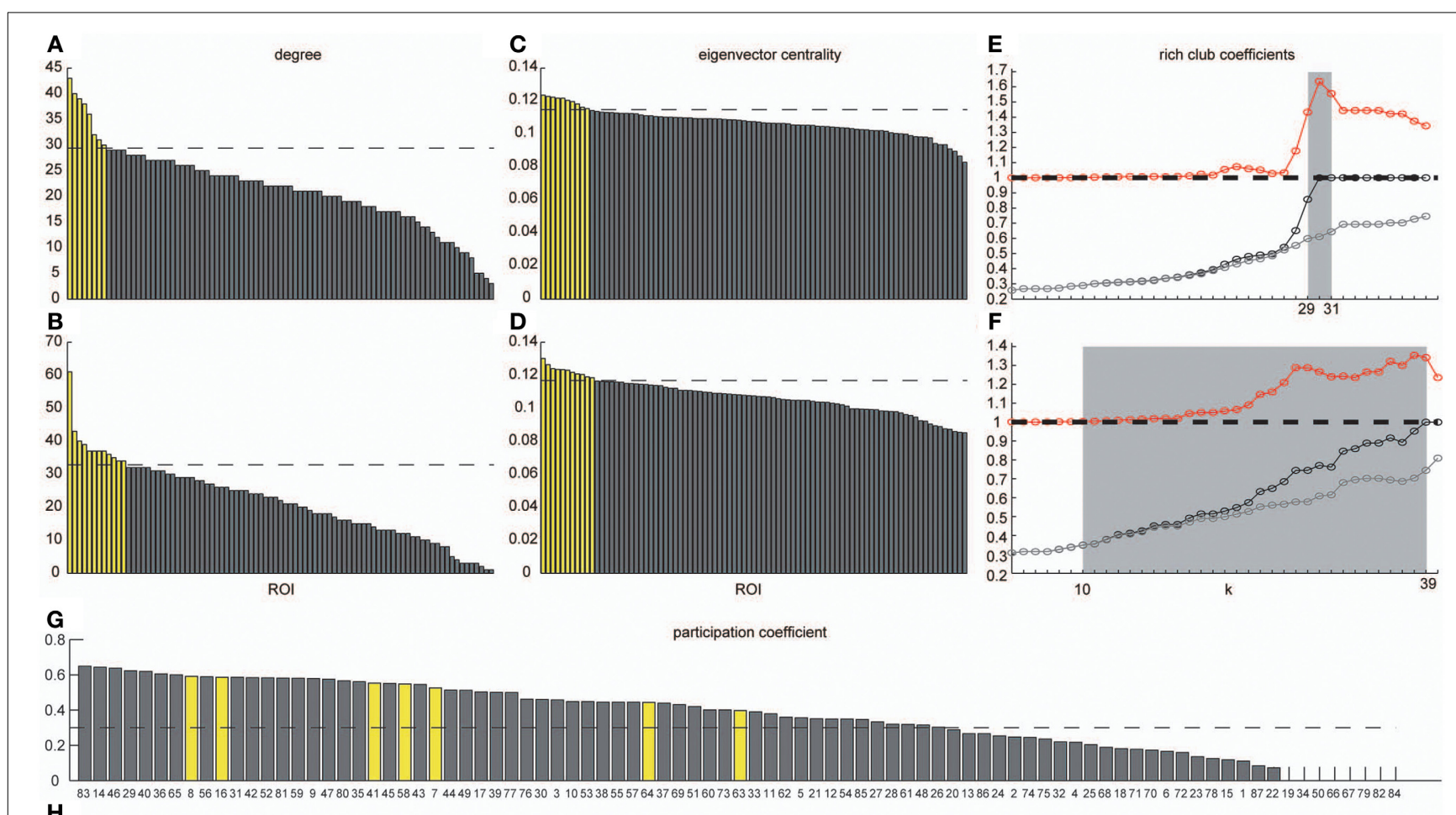

H
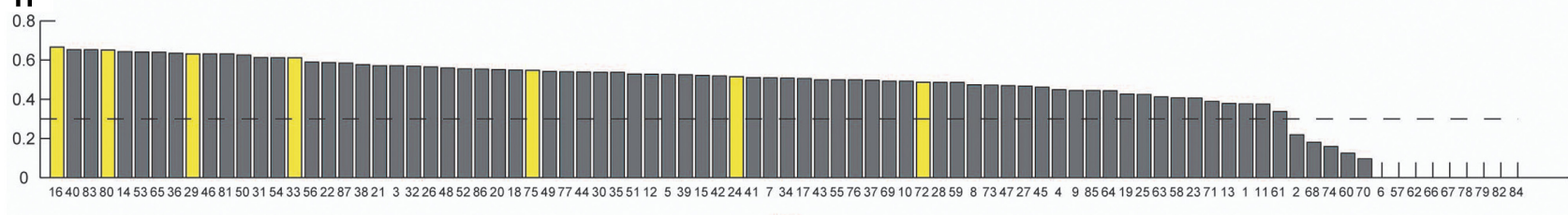

ROI

FIGURE 6 | Degrees, eigenvector centrality, rich-club coefficients and participation coefficients. Degrees and eigenvector centralities of all nodes were calculated in the group-level network estimated in the VLF and LF. (A) Shows the distribution of degree estimated in the VLF, and (B) shows the distribution of degree estimated in the LF. (C) Corresponds to the distribution of eigenvector centrality estimated in the VLF, and (D) corresponds to the distribution of eigenvector centrality estimated in the LF. Dashed lines express the mean plus one standard deviation. Yellow bars represent nodal metrics above the threshold (the mean plus one standard deviation), and gray bars indicate those below the threshold. We also calculated rich-club coefficients in group-level frequency-specific networks (see "Rich-club detection"). Black curves correspond to $\Phi(k)$, gray curves correspond to $\Phi_{\text {meanrand }}(k)$, and red curves correspond to $\Phi_{\text {normalized }}(k)$. In both $(\mathbf{E}, \mathbf{F})$, there is a tendency for $\Phi(k)$ to increase with $k$ at a higher rate than $\Phi_{\text {meanrand }}(k)$. Ranges of $k$, where $\Phi(k)$ became significantly higher than $\Phi_{\text {meanrand }}(k)$, are highlighted by a gray background. $(\mathbf{G}, \mathbf{H})$ Show the participation coefficients of all ROIs in the VLF and LF, respectively. Yellow bars represent the coefficients of hubs in each frequency band. Broken lines indicate 0.3 , which is the boundary between provincial and connector hubs (see Materials and Methods). between detected communities. Using the optimal algorithm for detecting communities in two networks that consist of the same node set, but are connected by two different connection patterns, we could measure and compare how clearly the community structures were present in both networks. In the current study, the same communities were found, but there was a significant difference in modularity between frequency-specific networks in the VLF and LF. These results clearly demonstrate that separation between communities in the network in the VLF was significantly stronger than that in the LF.

The observed hubs were not sensitive to the algorithm selection in the current study. We identified hub regions using two graph measures that characterize different aspects of nodes in a network: the nodal degree and the eigenvector centrality. The nodal degree was defined as the number of connectivity in the current study, and the eigenvector centrality quantified the influence of a node in a network. Individual application of the two measures could potentially detect different hubs in the same network. We therefore defined hubs using both criteria, meaning that our findings were not sensitive to the selection process. We found hub nodes in both networks in the VLF and LF, suggesting that these frequency-specific networks consistently show network structures in which a small set of highly connected and highly influential nodes was present.

"Rich-club" organization is a network structure characterized by the presence of highly interconnected hubs in a network. It was recently demonstrated that rich-club organization is present in structural networks of the nervous system of humans, monkeys, cats, and C. elegans (van den Heuvel and Sporns, 2011; Harriger et al., 2012; Towlson et al., 2013; de Reus and van den 
Table 2 | List of hubs identified in 2 frequency-specific networks.

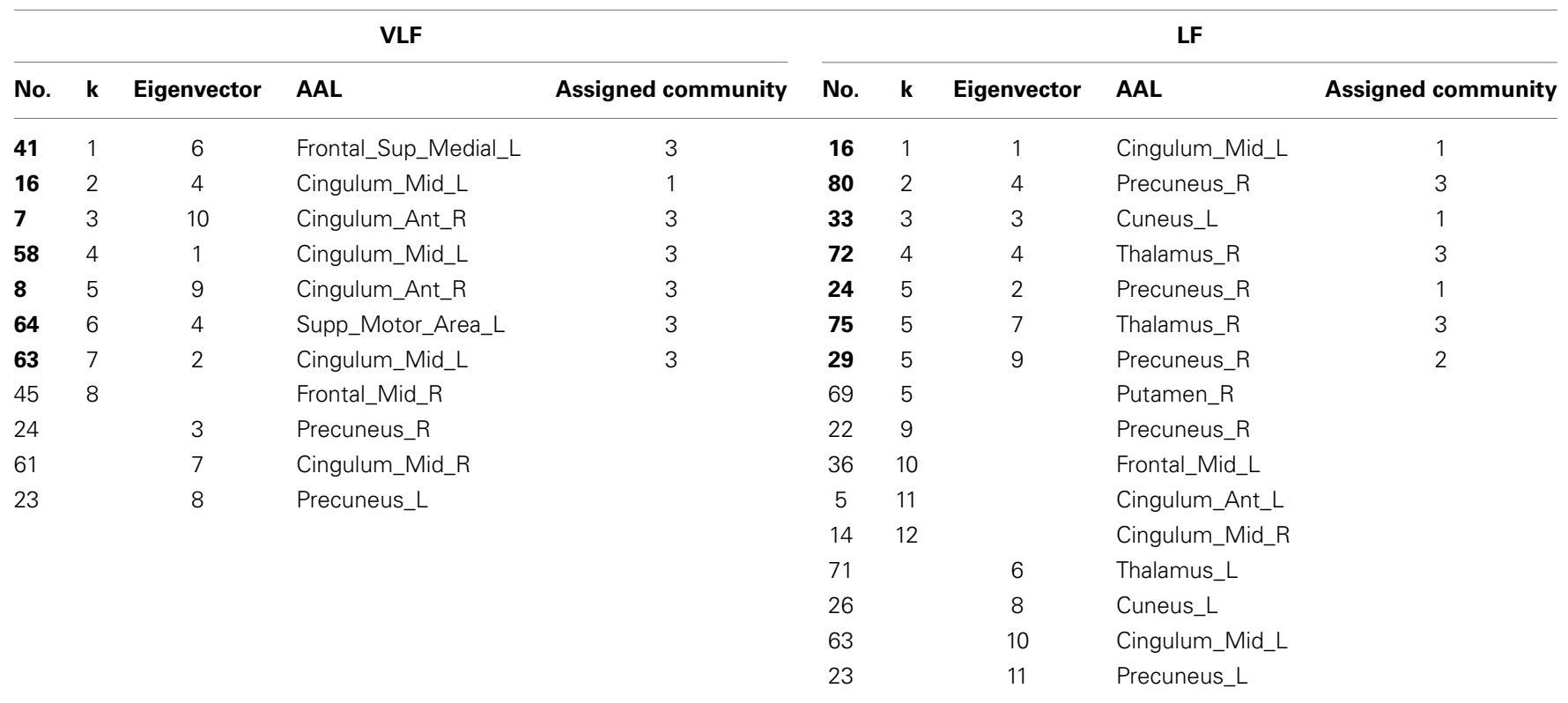

The hubs identified in each network are shown. To identify hub regions, we calculated the group means of the coherence values and the estimated group-averaged VLFN and LFN. We selected a sparsity of 0.25 as the threshold value, because all networks become connected in graphs only using this value. Then, a hub was defined as a node satisfying the following two conditions: first, the nodal degree was higher than the mean plus the standard deviation of the degree distribution; and second, the eigenvector centrality that was measured in a weighted non-thresholded network was higher than the mean plus the standard deviation of the distribution of the centrality. In the column assigned "No," bold type-face shows identified hubs, and normal type-face represents non-hub nodes that have a larger nodal degree or eigenvector centrality than the thresholds. The numbers in column $k$ and the eigenvector indicate the ranks of the nodes for the above two conditions, respectively. Anatomical labels obtained from AAL are listed in column AAL. The numbers in the column assigned community indicate those of detected communities where hubs were assigned in each frequency-specific network.

Heuvel, 2013). Connections within the rich-club organization, which are referred to as "rich-club connections" in the present work, are thought not only to increase the efficiency of global functional integration by bypassing hubs, but also to make the network robust against attacks on the hubs (van den Heuvel and Sporns, 2011). Our findings showing that all of the detected hubs formed rich-club organizations in the current study supports the idea that, independent of frequency-bands, the FCN has a higherorder integration structure that might play a role in the functional integration of the network. Although spatial correspondence among regions of interest are obscure, parts of observed richclub regions such as the superior frontal, precuneus and thalamus (Figures 7C-F) appear to be included in the structural network reported by van den Heuvel and Sporns (2011). Notably, the rich-club regions in FCNs differed in a frequency-dependent manner within the FCNs of the VLF (Figures 7C,D) and LF (Figures 7E,F). Considering the fact that frequency-specific networks in the VLF and LF have different connection patterns, these diverse rich-club organizations might serve in global, but differential, functional integration specific to the timescales of brain activity.

Using a participation coefficient allowed us to estimate and classify the functional role of hubs in relationship to a community. This measure revealed that all of the hubs in the current study could be classified as "connectors" (Figures 6G,H, and Table 2); this suggests that hubs promote functional integration among the COS, FPS, and DMS in both the VLF and LF. All of the frequency-specific hubs in the VLF were located within the COS, suggesting that it might act as an integration center among these three functional systems in the VLF. By contrast, frequency-specific hubs in the LF were detected in all functional systems (the COS, FPS, and DMS), suggesting that there is no such integration center in the FCN of the LF. Conversely, functional integration might occur directly among all communities within this frequency band. Furthermore, modularity in the LF was significantly lower than that in the VLF-that is, there was more connectivity across boundaries of communities in the FCN for the LF. This supports the idea that direct functional integration among communities can occur more easily in the LF than in the VLF through distributed hubs over all communities and direct connectivity among communities.

The relationship between frequency-components of the fMRI signal and its function has recently been investigated. Some studies focused on the band-specific power of the fMRI signal. For example, Baria et al. (2011) divided fMRI signals into four separate frequency-bands $(0.01-0.05,0.05-0.10,0.10-0.15$, and $0.15-0.20 \mathrm{~Hz}$ ) and found opposite task-induced shifts in the mean of the whole-brain power between the lowest $(0.01-0.05 \mathrm{~Hz})$ and second lowest bands $(0.05-0.10 \mathrm{~Hz})$. Other groups decomposed the frequency-band into $0.01-0.027$ and $0.027-0.08 \mathrm{~Hz}$ and reported frequency-specific relationships of band-limited amplitudes with personality traits (Wei et al., 2014), or among subjects with brain disorders, including amnestic mild cognitive impairment (Han et al., 2011) and schizophrenia (Yu et al., 2014). 


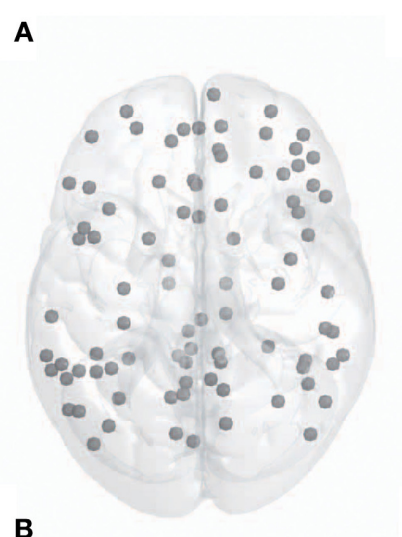

C

L

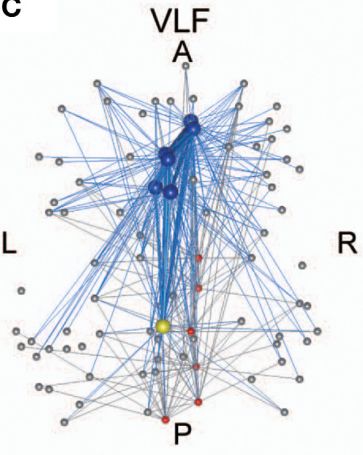

D 64: Supp_Motor_Area_L

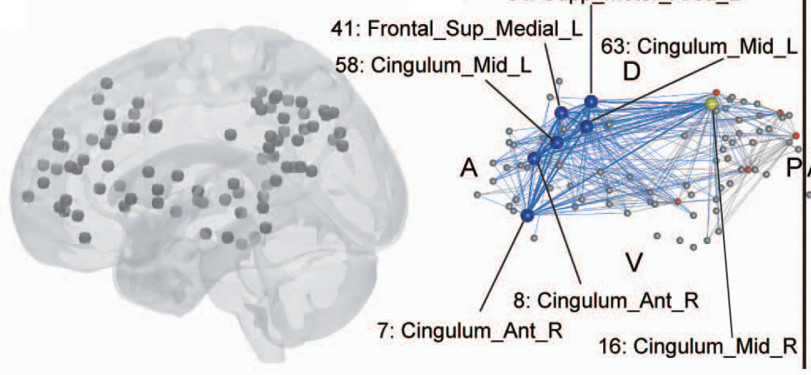

E

R $\mathrm{L}$
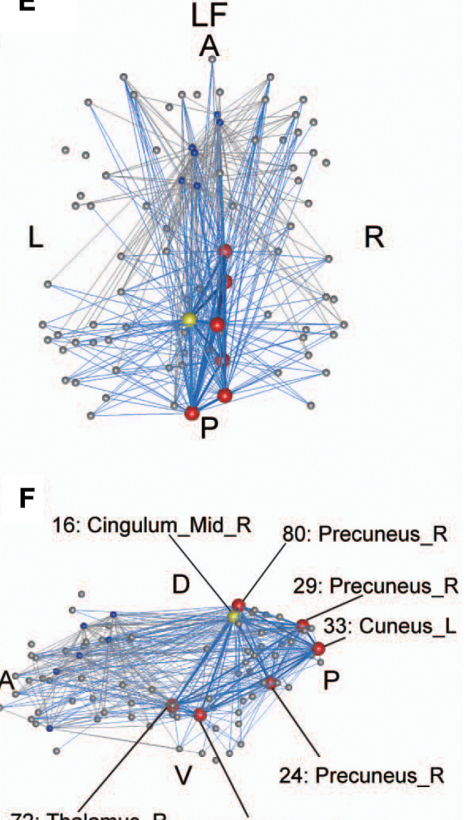

72: Thalamus_R 75: Thalamus_R

G

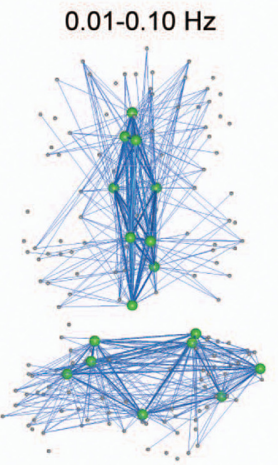

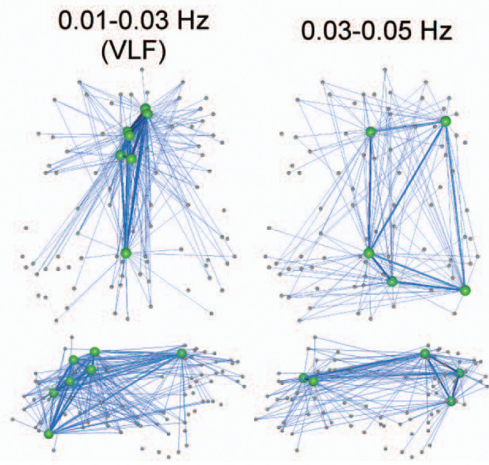

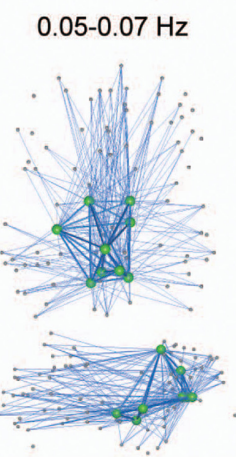

$0.07-0.09 \mathrm{~Hz}$

(LF)

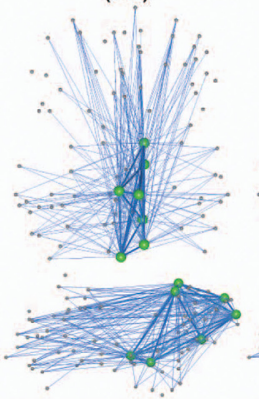

$0.09-0.11 \mathrm{~Hz}$

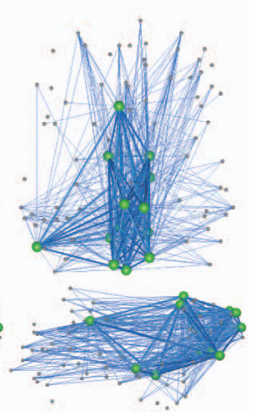

FIGURE 7 | Anatomical perspective of hub regions. (A,B) Eighty-seven ROls used in the current study are displayed on a surface rendering of the brain using MATcro software distributed by http://www.mccauslandcenter.sc.edu/ CRNL/tools/surface-rendering-with-matlab. Hub regions in frequency-specific networks were the seven highest degree nodes in each frequency band (C-F). The yellow node is a hub that is consistently identified in both the VLF (C,D) and the LF (E,F). The blue nodes are hubs identified only in the VLF, and the red nodes are those identified only in the LF. Hubs are represented by large spheres. Blue lines indicate functional connectivity with hubs within the frequency band, and gray lines represent functional connectivity with hubs within the other band. When $k=30$ for $A^{g} \mathrm{VLF}$ and $k=30$ for $A^{g}$ LF is selected, rich-club organizations are formed with hub regions. Bold lines indicate connections among rich-club nodes, showing dense interconnections. The numbers correspond to those in Table 1. Anatomical labels were selected using AAL. The abbreviations represent the direction in the brain: A, anterior; P, posterior; L, left; R, right; D, dorsal; V, ventral. (G) Hub regions identified within one wide frequency band $(0.01-0.10 \mathrm{~Hz})$ and five narrow frequency bands (0.01-0.03 [VLF], 0.03-0.05, 0.05-0.07, 0.07-0.09 [LF], and $0.09-0.11 \mathrm{~Hz}$ ). All hub nodes are represented with large green colored spheres. The attributes of the lines are the same as described above. 
While these studies focused on the relationship between amplitudes of fMRI signals and functions, Lohmann et al. (2010) found that some regions, including the precuneus and thalamus, show frequency-specific changes of voxel-wise eigenvector centrality between states of hunger and satiety. Furthermore, our findings demonstrated that there are frequency-specific network organizations with distinct topologies in the FCN. Since our data sets were obtained during a resting state, we cannot interpret our results in terms of tasks or behaviors. However, we speculate that differences between network topologies reflect frequency-specific dominance of functional segregation and integration. Although this proposal remains speculative, future investigations exploring the frequency-specific changes of network topology with several tasks and brain states would provide important clues for clarifying the spectral properties of brain functions.

There are two limitations of the methods used in our study. First, non-neural fluctuations included in hemodynamic signals might have affected the current results. It has been demonstrated that hemodynamic signals include signal fluctuations with non-neural physiological origins such as respiratory and cardiac pulsations (Lowe et al., 1998; Bhattacharyya and Lowe, 2004), changes in respiratory and cardiac rates (Wise et al., 2004; Birn et al., 2006; Shmueli et al., 2007), blood pressure (Katura et al., 2006), and changes in vascular tone for cerebral autoregulation (Lagopoulos et al., 2006), and vasomotion (Aalkjaer et al., 2011). Coherence due to the respiratory and cardiac pulsations had peaks out of $0.01-0.10 \mathrm{~Hz}$ ([respiratory: $\sim 0.3 \mathrm{~Hz}]$ and [cardiac: $\sim 1 \mathrm{~Hz}$ ]) (Figures 3D,E). Because the sampling rate of fMRI is not sufficiently high, the functional connectivity can be biased by aliasing of these confounds with higher frequencies. By contrast, using NIRS with a sampling rate of $10 \mathrm{~Hz}$ revealed that frequency characteristics of functional connectivity are not due to aliasing of noise. However, the frequency ranges of some of these non-neural fluctuations, such as changes in respiratory and cardiac rates, blood pressure, and vasomotion, were included in the hemodynamic signals fluctuating within $0.01-0.10 \mathrm{~Hz}$. Moreover, it is possible that signal fluctuations with non-neural physiological origins are contained uniformly in gray matter voxels (Desjardins et al., 2001; Greicius et al., 2003; Macey et al., 2004). Bias in the estimation of functional connectivity due to physiological confounds can be avoided by using methods that simultaneously record physiological data with fMRI signals (Glover et al., 2000) and/or by exploiting inherent information in the resting state fMRI data (Fox et al., 2005; Chang and Glover, 2009; Anderson et al., 2011; Chai et al., 2012). In the current study, we applied the PSTCor method, which uses only resting state fMRI data sets for correction, in order to factor out non-neural fluctuations to increase confidence in the results of functional connectivity (Anderson et al., 2011). However, although using this method for physiological correction can suppress over-estimation of functional connectivity, it might cause under-estimation because variations in physiological regulations might be correlated with neural activities (Murphy et al., 2013). For example, emotional arousal and activity levels of the autonomic nervous system are indicated by variability of heart rate (Macefield, 2009). Furthermore, it is unclear whether there are regional specific physiological confounds. Because the above-mentioned correction cannot account for all such signals, other methods will be required. Moreover, in comparison to other physiological confounds, the contribution of vasomotion to hemodynamic signals is poorly understood (Murphy et al., 2013). For example, it is still unclear whether vasomotion has an effect on hemodynamics independently of other physiological origins (Morita-Tsuzuki et al., 1992; Hudetz et al., 1998; Biswal and Kannurpatti, 2009). Further studies regarding the relationship between physiology and hemodynamic signals are needed to ensure certainty of results concerning functional connectivity.

Second, state-dependency of functional connectivity must be considered. Although most studies of functional connectivity collect 5-11 min of resting state fMRI data, some have reported non-stationary characteristics using similar length resting state data sets (Chang and Glover, 2010; Hutchison et al., 2012). To characterize the length of time required to acquire reproducible functional connectivity measurements, much effort has been focused on test-retest reliability of functional connectivity maps. Earlier studies showed that the strength of functional connectivity becomes stable when using data sets longer than $7 \mathrm{~min}$ (Shehzad et al., 2009; Van Dijk et al., 2010; Thomason et al., 2011; Braun et al., 2012; Li et al., 2012). However, a recent investigation into this issue with a longer scan length $(27 \mathrm{~min})$ than that in previous studies demonstrated that improvements in test-retest reliability plateaued around $12-16 \mathrm{~min}$ for intra-session comparisons and at 8-12 min for inter-session comparisons (Birn et al., 2013). Therefore, to achieve reliable results, we need to acquire resting state data for longer than $16 \mathrm{~min}$. Because we used $20 \mathrm{~min}$ resting state data to calculate functional connectivity in the current study, we were able to derive our results free from this bias. However, it should be noted that although the highest test-retest reliabilities could be provided by the scan length used in the current study, the degree of reliability for inter-session variability is lower than that of intra-session variability (Birn et al., 2013). Further investigation into the state-dependency of the FCN would provide further insights.

Theoretical views on the anatomical wiring of the brain suggest that it has a fundamental characteristic of reconciling the apparently opposing demands of local segregation and global integration of information (Felleman and Van Essen, 1991; Tononi et al., 1994). This attribute has also been demonstrated to exist in the network structure of functional brain organization (Sporns, 2013). Our current results suggest that the spatial order of the brain can vary by measuring it with different timescales. Indeed, we demonstrated the co-existence of two functional brain organizations in a frequency-dependent manner. Since different network topologies might contribute to different brain functions, the present study promotes further investigation into the relationship between frequency-specific network topologies and the timescales of human behavior.

\section{ACKNOWLEDGMENTS}

We thank Kayo Asakawa for providing administrative assistance. We are grateful to Moe Kato for providing assistance in experiments. We thank Dr. Toru Nakamura and Dr. Masanori Shimono for helpful comments on analytical procedures. This work was partly supported by Grants-in-Aid for the Japan Society for 
the Promotion of Science (JSPS) Fellows 2311065 (to Shuntaro Sasai), for Young Scientists (S) 20670001 (to Gentaro Taga), and for Challenging Exploratory Research 23650224 (to Hiroki C. Tanabe). In addition, it was partly supported by the Ministry of Education, Culture, Sports, Science and Technology in Japan (MEXT) for Scientific Research (S) 21220005 (to Norihiro Sadato), and Scientific Research on Innovative Areas 22101007 (to Hiroki C. Tanabe). A part of this study is the result of "Development of biomarker candidates for social behavior" carried out under the Strategic Research Program for Brain Science by MEXT.

\section{SUPPLEMENTARY MATERIAL}

The Supplementary Material for this article can be found online at: http://www.frontiersin.org/journal/10.3389/fnhum. 2014.01022/abstract

\section{REFERENCES}

Aalkjaer, C., Boedtkjer, D., and Matchkov, V. (2011). Vasomotion-what is currently thought? Acta Physiol. (Oxf.) 202, 253-269. doi: 10.1111/j.17481716.2011.02320.x

Achard, S., and Bullmore, E. (2007). Efficiency and cost of economical brain functional networks. PLoS Comput. Biol. 3:e17. doi: 10.1371/journal.pcbi.0030017

Achard, S., Salvador, R., Whitcher, B., Suckling, J., and Bullmore, E. (2006). A resilient, low-frequency, small-world human brain functional network with highly connected association cortical hubs. J. Neurosci. 26, 63-72. doi: 10.1523/JNEUROSCI.3874-05.2006

Anderson, J. S., Druzgal, T. J., Lopez-Larson, M., Jeong, E. K., Desai, K., and Yurgelun-Todd, D. (2011). Network anticorrelations, global regression, and phase-shifted soft tissue correction. Hum. Brain Mapp. 32, 919-934. doi: 10.1002/hbm.21079

Baria, A. T., Baliki, M. N., Parrish, T., and Apkarian, A. V. (2011). Anatomical and functional assemblies of brain BOLD oscillations. J. Neurosci. 31, 7910-7919. doi: 10.1523/JNEUROSCI.1296-11.2011

Bassett, D. S., Bullmore, E., Verchinski, B. A., Mattay, V. S., Weinberger, D. R., and Meyer-Lindenberg, A. (2008). Hierarchical organization of human cortical networks in health and schizophrenia. J. Neurosci. 28, 9239-9248. doi: 10.1523/JNEUROSCI.1929-08.2008

Benjamini, Y., and Yekutieli, D. (2001). The control of the false discovery rate in multiple testing under dependency. Ann. Stat. 29, 1165-1188. doi: 10.1214/aos/ 1013699998

Bhattacharyya, P. K., and Lowe, M. J. (2004). Cardiac-induced physiologic noise in tissue is a direct observation of cardiac-induced fluctuations. Magn. Reson. Imaging 22, 9-13. doi: 10.1016/j.mri.2003.08.003

Birn, R. M., Diamond, J. B., Smith, M. A., and Bandettini, P. A. (2006). Separating respiratory-variation-related fluctuations from neuronalactivity-related fluctuations in fMRI. Neuroimage 31, 1536-1548. doi: 10.1016/j.neuroimage.2006.02.048

Birn, R. M., Molloy, E. K., Patriat, R., Parker, T., Meier, T. B., Kirk, G. R., et al. (2013). The effect of scan length on the reliability of resting-state fMRI connectivity estimates. Neuroimage 83, 550-558. doi: 10.1016/j.neuroimage.2013. 05.099

Biswal, B. B., and Kannurpatti, S. S. (2009). Resting-state functional connectivity in animal models: modulations by exsanguination. Methods Mol. Biol. 489, 255-274. doi: 10.1007/978-1-59745-543-5_12

Biswal, B., Yetkin, F. Z., Haughton, V. M., and Hyde, J. S. (1995). Functional connectivity in the motor cortex of resting human brain using echo-planar MRI. Magn. Reson. Med. 34, 537-541. doi: 10.1002/mrm.1910340409

Blondel, V., Guillaume, J. L., Lambiotte, R., and Lefebvre, E. (2008). Fast unfolding of communities in large networks. J. Stat. Mech. 2008:P10008. doi: 10.1088/1742-5468/2008/10/P10008

Braun, U., Plichta, M. M., Esslinger, C., Sauer, C., Haddad, L., Grimm, O., et al. (2012). Test-retest reliability of resting-state connectivity network characteristics using fMRI and graph theoretical measures. Neuroimage 59, 1404-1412. doi: 10.1016/j.neuroimage.2011.08.044
Buckner, R. L., Sepulcre, J., Talukdar, T., Krienen, F. M., Liu, H., Hedden, T., et al. (2009). Cortical hubs revealed by intrinsic functional connectivity: mapping, assessment of stability, and relation to Alzheimer's disease. J. Neurosci. 29, 1860-1873. doi: 10.1523/JNEUROSCI.5062-08.2009

Butts, C. T. (2009). Revisiting the foundations of network analysis. Science 325, 414-416. doi: 10.1126/science.1171022

Cecchi, G. A., Rao, A. R., Centeno, M. V., Baliki, M., Apkarian, A. V., and Chialvo, D. R. (2007). Identifying directed links in large scale functional networks: application to brain fMRI. BMC Cell Biol. 8(Suppl. 1):S5. doi: 10.1186/14712121-8-S1-S5

Chai, X. J., Castanon, A. N., Ongur, D., and Whitfield-Gabrieli, S. (2012). Anticorrelations in resting state networks without global signal regression. Neuroimage 59, 1420-1428. doi: 10.1016/j.neuroimage.2011.08.048

Chang, C., and Glover, G. H. (2009). Effects of model-based physiological noise correction on default mode network anti-correlations and correlations. Neuroimage 47, 1448-1459. doi: 10.1016/j.neuroimage.2009.05.012

Chang, C., and Glover, G. H. (2010). Time-frequency dynamics of restingstate brain connectivity measured with fMRI. Neuroimage 50, 81-98. doi: 10.1016/j.neuroimage.2009.12.011

Colizza, V., Flammini, A., Serrano, M. A., and Vespignani, A. (2006). Detecting rich- club ordering in complex networks. Nat. Phys. 2, 110-115. doi: 10.1038/nphys209

Cordes, D., Haughton, V. M., Arfanakis, K., Carew, J. D., Turski, P. A., Moritz, C. H., et al. (2001). Frequencies contributing to functional connectivity in the cerebral cortex in "resting-state" data. Am. J. Neuroradiol. 22, 1326-1333.

Crossley, N. A., Mechelli, A., Vértes, P. E., Winton-Brown, T. T., Patel, A. X., Ginestet, C. E., et al. (2013). Cognitive relevance of the community structure of the human brain functional coactivation network. Proc. Natl. Acad. Sci. U.S.A. 110, 11583-11588. doi: 10.1073/pnas.1220826110

Curtis, C. E., and D'Esposito, M. (2003). Persistent activity in the prefrontal cortex during working memory. Trends Cogn Sci. 7, 415-423. doi: 10.1016/S13646613(03)00197-9

de Reus, M. A., and van den Heuvel, M. P. (2013). Rich club organization and intermodule communication in the cat connectome. J. Neurosci. 33, 12929-12939. doi: 10.1523/JNEUROSCI.1448-13.2013

Deshpande, G., Santhanam, P., and Hu, X. (2011). Instantaneous and causal connectivity in resting state brain networks derived from functional MRI data. Neuroimage 54, 1043-1052. doi: 10.1016/j.neuroimage.2010.09.024

Desjardins, A. E., Kiehl, K. A., and Liddle, P. F. (2001). Removal of confounding effects of global signal in functional MRI analyses. Neuroimage 13, 751-758. doi: 10.1006/nimg.2000.0719

Dosenbach, N. U., Fair, D. A., Miezin, F. M., Cohen, A. L., Wenger, K. K., Dosenbach, R. A., et al. (2007). Distinct brain networks for adaptive and stable task control in humans. Proc. Natl. Acad. Sci. U.S.A. 104, 11073-11078. doi: 10.1073/pnas.0704320104

Dosenbach, N. U., Nardos, B., Cohen, A. L., Fair, D. A., Power, J. D., Church, J. A., et al. (2010). Prediction of individual brain maturity using fMRI. Science 329, 1358-1361. doi: 10.1126/science.1194144

Dosenbach, N. U., Visscher, K. M., Palmer, E. D., Miezin, F. M., Wenger, K. K., Kang, H. C., et al. (2006). A core system for the implementation of task sets. Neuron 50, 799-812. doi: 10.1016/j.neuron.2006.04.031

Eguíluz, V. M., Chialvo, D. R., Cecchi, G. A., Baliki, M., and Apkarian, A. V. (2005). Scale-free brain functional networks. Phys. Rev. Lett. 94:018102. doi: 10.1103/PhysRevLett.94.018102

Felleman, D. J., and Van Essen, D. C. (1991). Distributed hierarchical processing in the primate cerebral cortex. Cereb. Cortex 1, 1-47. doi: 10.1093/cercor/1.1.1

Fornito, A., Harrison, B. J., Zalesky, A., and Simons, J. S. (2012). Competitive and cooperative dynamics of large-scale brain functional networks supporting recollection. Proc. Natl. Acad. Sci. U.S.A. 109, 12788-12793. doi: 10.1073/pnas.1204185109

Fox, M. D., and Raichle, M. E. (2007). Spontaneous fluctuations in brain activity observed with functional magnetic resonance imaging. Nat. Rev. Neurosci. 8, 700-711. doi: 10.1038/nrn2201

Fox, M. D., Snyder, A. Z., Vincent, J. L., Corbetta, M., Van Essen, D. C., and Raichle, M. E. (2005). The human brain is intrinsically organized into dynamic, anticorrelated functional networks. Proc. Natl. Acad. Sci. U.S.A. 102, 9673-9678. doi: 10.1073/pnas.0504136102

Glover, G. H., Li, T. Q., and Ress, D. (2000). Image-based method for retrospective correction of physiological motion effects in fMRI: RETROICOR. Magn. 
Reson. Med. 44, 162-167. doi: 10.1002/1522-2594(200007)44:1<162::AIDMRM23>3.0.CO;2-E

Grayson, D. S., Ray, S., Carpenter, S., Iyer, S., Dias, T. G. C., Stevens, C., et al. (2014). Structural and Functional Rich Club Organization of the Brain in Children and Adults. PLoS ONE 9:e88297. doi: 10.1371/journal.pone.0088297

Greicius, M. D., Krasnow, B., Reiss, A. L., and Menon, V. (2003). Functional connectivity in the resting brain: a network analysis of the default mode hypothesis. Proc. Natl. Acad. Sci. U.S.A. 100, 253-258. doi: 10.1073/pnas.01350 58100

Guimera, R., and Amaral, L. A. N. (2005). Functional cartography of complex metabolic networks. Nature 433, 895-900. doi: 10.1038/nature03288

Gusnard, D. A., Akbudak, E., Shulman, G. L., and Raichle, M. E. (2001). Medial prefrontal cortex and self-referential mental activity: relation to a default mode of brain function. Proc. Natl. Acad. Sci. U.S.A. 98, 4259-4264. doi: 10.1073/pnas.071043098

Hagmann, P., Cammoun, L., Gigandet, X., Meuli, R., Honey, C. J., Wedeen, V. J., et al. (2008). Mapping the structural core of human cerebral cortex. PLoS Biol. 6:e159. doi: 10.1371/journal.pbio.0060159

Han, U., Wang, J., Zhao, Z., Min, B., Lu, J., Li, K., et al. (2011). Frequencydependent changes in the amplitude of low-frequency fluctuations in amnestic mild cognitive impairment: a resting-state fMRI study. Neuroimage 55, 287-295 doi: 10.1016/j.neuroimage.2010.11.059

Harriger, L., van den Heuvel, M. P., and Sporns, O. (2012). Rich club organization of macaque cerebral cortex and its role in network communication. PLoS ONE 7:e46497. doi: 10.1371/journal.pone.0046497

Hayasaka, S., and Laurienti, P. J. (2010). Comparison of characteristics between region-and voxel-based network analyses in resting-state fMRI data. Neuroimage 50, 499-508. doi: 10.1016/j.neuroimage.2009.12.051

He, B. J. (2011). Scale-free properties of the functional magnetic resonance imaging signal during rest and task. J. Neurosci. 31, 13786-13795. doi: 10.1523/JNEUROSCI.2111-11.2011

Honey, C. J., Kötter, R., Breakspear, M., and Sporns, O. (2007). Network structure of cerebral cortex shapes functional connectivity on multiple time scales. Proc. Natl. Acad. Sci. U.S.A. 104, 10240-10245. doi: 10.1073/pnas.0701519104

Hudetz, A. G., Biswal, B. B., Shen, H., Lauer, K. K., and Kampine, J. P. (1998). Spontaneous fluctuations in cerebral oxygen supply. An introduction. Adv. Exp. Med. Biol. 454, 551-559. doi: 10.1007/978-1-4615-4863-8_66

Hutchison, R. M., Womelsdorf, T., Gati, J. S., Everling, S., and Menon, R. S. (2012). Resting-state networks show dynamic functional connectivity in awake humans and anesthetized macaques. Hum. Brain Mapp. 34, 2154-2177. doi: 10.1002/hbm. 22058

Katura, T., Tanaka, N., Obata, A., Sato, H., and Maki, A. (2006). Quantitative evaluation of interrelations between spontaneous low-frequency oscillations in cerebral hemodynamics and systemic cardiovascular dynamics. Neuroimage 31, 1592-1600. doi: 10.1016/j.neuroimage.2006.02.010

Kerns, J. G., Cohen, J. D., MacDonald, A. W. 3rd., Cho, R. Y., Stenger, V. A., and Carter, C. S. (2004). Anterior cingulate conflict monitoring and adjustments in control. Science 303, 1023-1026. doi: 10.1126/science.1089910

Kuncheva, L., and Hadjitodorov, S. (2004). Using diversity in cluster ensembles. IEEE Int. Conf. Syst. Man Cybern. 2, 1214-1219. doi: 10.1109/ICSMC.2004. 1399790

Lagopoulos, J., Malhi, G. S., Cahill, A. M., Lang, E. W., Mudalier, Y., Dorsch, N., et al. (2006). Cerebrovascular autoregulation as a neuroimaging tool. Acta Neuropsychiatr. 18, 100-104. doi: 10.1111/j.1601-5215.2006.00133.x

Latora, V., and Marchiori, M. (2001). Efficient behavior of small-world networks. Phys. Rev. Lett. 87, 198701. doi: 10.1103/PhysRevLett.87.198701

Li, Z., Kadivar, A., Pluta, J., Dunlop, J., and Wang, Z. (2012). Test-retest stability analysis of resting brain activity revealed by blood oxygen level-dependent functional MRI. J. Magn. Reson. Imaging 36, 344-354. doi: 10.1002/jmri. 23670

Lohmann, G., Margulies, D. S., Horstmann, A., Pleger, B., Lepsien, J., Goldhahn, D., et al. (2010). Eigenvector centrality mapping for analyzing connectivity patterns in fMRI data of the human brain. PLOS ONE 5:e10232. doi: 10.1371/journal.pone.0010232

Lowe, M. J., Mock, B. J., and Sorenson, J. A. (1998). Functional connectivity in single and multislice echoplanar imaging using resting-state fluctuations. Neuroimage 7, 119-132. doi: 10.1006/nimg.1997.0315

Macefield, V. G. (2009). Developments in autonomic research: a review of the latest literature. Clin. Auton. Res. 19, 193-196. doi: 10.1007/s10286-009-0024-3
Macey, P. M., Macey, K. E., Kumar, R., and Harper, R. M. (2004). A method for removal of global effects from fMRI time series. Neuroimage 22, 360-366. doi: 10.1016/j.neuroimage.2003.12.042

McAuley, J. J., da Fontoura Costa, L., and Caetano, T. S. (2007). Rich-club phenomena across complex network hierachies. Appl. Phys. Lett. 91:084103. doi: $10.1063 / 1.2773951$

Menon, V., Adleman, N. E., White, C. D., Glover, G. H., and Reiss, A. L. (2001). Error-related brain activation during a Go/NoGo response inhibition task. Hum. Brain Mapp. 12, 131-143. doi: 10.1002/10970193(200103)12:3<131::AID-HBM1010>3.0.CO;2-C

Menon, V., and Uddin, L. Q. (2010). Saliency, switching, attention and control: a network model of insula function. Brain Struct. Funct. 214, 655-667. doi: 10.1007/s00429-010-0262-0

Meunier, D., Lambiotte, R., Fornito, A., Ersche, K. D., and Bullmore, E. T. (2009). Hierarchical modularity in human brain functional networks. Front Neuroinform. 3:37. doi: 10.3389/neuro.11.037.2009

Morita-Tsuzuki, Y., Bouskela, E., and Hardebo, J. E. (1992). Vasomotion in the rat cerebral micro-circulation recorded by laser-Doppler flowmetry. Acta Physiol. Scand. 146, 431-439. doi: 10.1111/j.1748-1716.1992.tb09444.x

Murphy, K., Birn, R. M., and Bandettini, P. A. (2013). Resting-state fMRI confounds and cleanup. Neuroimage 80, 349-359. doi: 10.1016/j.neuroimage.2013.04.001

Murphy, K., Birn, R. M., Handwerker, D. A., Jones, T. B., and Bandettini, P. A. (2009). The impact of global signal regression on resting state correlations: are anti-correlated networks introduced? Neuroimage 44, 893-905. doi: 10.1016/j.neuroimage.2008.09.036

Newman, M. E. J. (2004). Fast algorithm for detecting community structure in networks. Phys. Rev. E 69:066133. doi: 10.1103/PhysRevE.69.066133

Newman, M. E. J. (2006). Modularity and community structure in networks. Proc. Natl. Acad. Sci. U.S.A. 103, 8577-8582. doi: 10.1073/pnas.0601602103

Obrig, H., Neufang, M., Wenzel, R., Kohl, M., Steinbrink, J., Einhaupl, K., et al. (2000). Spontaneous low frequency oscillations of cerebral hemodynamics and metabolism in human adults. Neuroimage 12, 623-639. doi: 10.1006/nimg.2000.0657

Power, J. D., Cohen, A. L., Nelson, S. M., Wig, G. S., Barnes, K. A., Church, J. A., et al. (2011). Functional network organization of the human brain. Neuron 72, 665-678. doi: 10.1016/j.neuron.2011.09.006

Power, J. D., Schlaggar, B. L., Lessov-Schlaggar, C. N., and Petersen, S. E. (2013). Evidence for hubs in human functional brain networks. Neuron 79, 798-813. doi: 10.1016/j.neuron.2013.07.035

Raichle, M., MacLeod, A., Snyder, A., Powers, W., Gusnard, D., and Shulman, G. (2001). A default mode of brain function. Proc. Natl. Acad. Sci. U.S.A. 98, 676-682. doi: 10.1073/pnas.98.2.676

Ridderinkhof, K. R., Ullsperger, M., Crone, E. A., and Nieuwenhuis, S. (2004). The role of the medial frontal cortex in cognitive control. Science 306, 443-447. doi: $10.1126 /$ science. 1100301

Rubinov, M., and Sporns, O. (2010). Complex network measures of brain connectivity: uses and interpretations. Neuroimage 52, 1059-1069. doi: 10.1016/j.neuroimage.2009.10.003

Salvador, R., Martinez, A., Pomarol-Clotet, E., Gomar, J., Vila, F., Sarro, S., et al (2008). A simple view of the brain through a frequency-specific functional connectivity measure. Neuroimage 39, 279-289. doi: 10.1016/j.neuroimage.2007.08. 018

Salvador, R., Martínez, A., Pomarol-Clotet, E., Sarró, S., Suckling, J., and Bullmore, E. (2007). Frequency based mutual information measures between clusters of brain regions in functional magnetic resonance imaging. Neuroimage 35, 83-88. doi: 10.1016/j.neuroimage.2006.12.001

Salvador, R., Suckling, J., Coleman, M. R., Pickard, J. D., Menon, D., and Bullmore, E. (2005a). Neurophysiological architecture of functional magnetic resonance images of human brain. Cereb. Cortex 15, 1332-1342. doi: 10.1093/cercor/bhi016

Salvador, R., Suckling, J., Schwarzbauer, C., and Bullmore, E. (2005b). Undirected graphs of frequency-dependent functional connectivity in whole brain networks. Philos. Trans. R. Soc. Lond. B. Biol. Sci. 360, 937-946. doi: 10.1098/rstb.2005.1645

Sasai, S., Homae, F., Watanabe, H., Sasaki, A. T., Tanabe, H. C., Sadato, N., et al. (2012). A NIRS-fMRI study of resting state network. Neuroimage 63, 179-193. doi: 10.1016/j.neuroimage.2012.06.011

Sasai, S., Homae, F., Watanabe, H., and Taga, G. (2011). Frequencyspecific functional connectivity in the brain during resting state revealed 
by NIRS. Neuroimage 56, 252-257. doi: 10.1016/j.neuroimage.2010. 12.075

Seeley, W. W., Menon, V., Schatzberg, A. F., Keller, J., Glover, G. H., Kenna, H., et al. (2007). Dissociable intrinsic connectivity networks for salience processing and executive control. J. Neurosci. 27, 2349-2356. doi: 10.1523/JNEUROSCI.558706.2007

Shehzad, Z., Kelly, A. M., Reiss, P. T., Gee, D. G., Gotimer, K., Uddin, L. Q., et al. (2009). The resting brain: unconstrained yet reliable. Cereb. Cortex 19, 2209-2229. doi: 10.1093/cercor/bhn256

Shmueli, K., van Gelderen, P., de Zwart, J. A., Horovitz, S. G., Fukunaga, M., Jansma, J. M., et al. (2007). Low-frequency fluctuations in the cardiac rate as a source of variance in the resting-state fMRI BOLD signal. Neuroimage 38, 306-320. doi: 10.1016/j.neuroimage.2007.07.037

Sporns, O. (2013). Network attributes for segregation and integration in the human brain. Curr. Opin. Neurobiol. 23, 162-171. doi: 10.1016/j.conb.2012.11.015

Sporns, O., Honey, C. J., and Kötter, R. (2007). Identification and classification of hubs in brain networks. PLoS ONE 2:e1049. doi: 10.1371/journal.pone.0001049

Spreng, R. N., Sepulcre, J., Turner, G. R., Stevens, W. D., and Schacter, D. L. (2013). Intrinsic architecture underlying the relations among the default, dorsal attention, and frontoparietal control networks of the human brain. J. Cogn. Neurosci. 25, 74-86. doi: 10.1162/jocn_a_00281

Sun, Y., Danila, B., Josicì, K., and Bassler, K. E. (2009). Improved community structure detection using a modified fine-tuning strategy. Europhys. Lett. 86, 28004. doi: 10.1209/0295-5075/86/28004

Thomason, M. E., Dennis, E. L., Joshi, A. A., Joshi, S. H., Dinov, I. D., Chang, C., et al. (2011). Resting-state fMRI can reliably map neural networks in children. Neuroimage 55, 165-175. doi: 10.1016/j.neuroimage.2010.11.080

Tomasi, D., and Volkow, N. D. (2011a). Association between functional connectivity hubs and brain networks. Cereb. Cortex 21, 2003-2013. doi: 10.1093/cercor/bhq268

Tomasi, D., and Volkow, N. D. (2011b). Functional connectivity hubs in the human brain. Neuroimage 57, 908-917. doi: 10.1016/j.neuroimage.2011.05.024

Tononi, G., Sporns, O., and Edelman, G. M. (1994). A measure for brain complexity: relating functional segregation and integration in the nervous system. Proc. Natl. Acad. Sci. U.S.A. 91, 5033-5037. doi: 10.1073/pnas.91.11.5033

Towlson, E. K., Vértes, P. E., Ahnert, S. E., Schafer, W. R., and Bullmore, E. T. (2013). The rich club of the C. elegans neuronal connectome. J. Neurosci. 33, 6380-6387. doi: 10.1523/JNEUROSCI.3784-12.2013

Tzourio-Mazoyer, N., Landeau, B., Papathanassiou, D., Crivello, F., Etard, O., Delcroix, N., et al. (2002). Automated anatomical labeling of activations in SPM using a macroscopic anatomical parcellation of the MNI MRI single-subject brain. Neuroimage 15, 273-289. doi: 10.1006/nimg.2001.0978

van den Heuvel, M., Mandl, R., and Hulshoff Pol, H. (2008a). Normalized cut group clustering of resting-state FMRI data. PLoS ONE 3:e2001. doi: 10.1371/journal.pone.0002001 van den Heuvel, M. P., and Sporns, O. (2011). Rich-club organization of the human connectome. J. Neurosci. 31, 15775-15786. doi: 10.1523/JNEUROSCI.353911.2011

van den Heuvel, M. P., Stam, C. J., Boersma, M., and Hulshoff Pol, H. E. (2008b). Small-world and scale-free organization of voxel-based resting-state functional connectivity in the human brain. Neuroimage 43, 528-539. doi: 10.1016/j.neuroimage.2008.08.010

Van Dijk, K. R., Hedden, T., Venkataraman, A., Evans, K. C., Lazar, S. W., and Buckner, R. L. (2010). Intrinsic functional connectivity as a tool for human connectomics: theory, properties, and optimization. J. Neurophysiol. 103, 297-321. doi: $10.1152 /$ jn.00783.2009

Wei, L., Duan, X., Zheng, C., Wang, S., Gao, Q., Zhang, Z., et al. (2014). Specific frequency bands of amplitude low-frequency oscillation encodes personality. Hum. Brain Mapp. 35, 331-339. doi: 10.1002/hbm.22176

Wise, R. G., Ide, K., Poulin, M. J., and Tracey, I. (2004). Resting fluctuations in arterial carbon dioxide induce significant low frequency variations in BOLD signal. Neuroimage 21, 1652-1664. doi: 10.1016/j.neuroimage.2003. 11.025

Wu, C. W., Gu, H., Lu, H., Stein, E. A., Chen, J. H., and Yang, Y. (2008). Frequency specificity of functional connectivity in brain networks. Neuroimage 42, 1047-1055. doi: 10.1016/j.neuroimage.2008.05.035

Yu, R., Chien, Y. L., Wang, H. L. S., Liu, C. M., Liu, C. C., Hwang, T. J., et al. (2014). Frequency-specific alternations in the amplitude of low-frequency fluctuations in schizophrenia. Hum. Brain Mapp. 35, 627-637. doi: 10.1002/hbm. 22203

Zuo, X. N., Di Martino, A., Kelly, C., Shehzad, Z. E., Gee, D. G., Klein, D. F., et al. (2010). The oscillating brain: complex and reliable. Neuroimage 49, 1432-1445. doi: 10.1016/j.neuroimage.2009.09.037

Conflict of Interest Statement: The authors declare that the research was conducted in the absence of any commercial or financial relationships that could be construed as a potential conflict of interest.

Received: 15 July 2014; accepted: 04 December 2014; published online: 22 December 2014.

Citation: Sasai S, Homae F, Watanabe H, Sasaki AT, Tanabe HC, Sadato N and Taga $G$ (2014) Frequency-specific network topologies in the resting human brain. Front. Hum. Neurosci. 8:1022. doi: 10.3389/fnhum.2014.01022

This article was submitted to the journal Frontiers in Human Neuroscience.

Copyright (c) 2014 Sasai, Homae, Watanabe, Sasaki, Tanabe, Sadato and Taga. This is an open-access article distributed under the terms of the Creative Commons Attribution License (CC BY). The use, distribution or reproduction in other forums is permitted, provided the original author $(s)$ or licensor are credited and that the original publication in this journal is cited, in accordance with accepted academic practice. No use, distribution or reproduction is permitted which does not comply with these terms. 